\title{
Intra-articular vs. intravenous administration: a meta-analysis of tranexamic acid in primary total knee arthroplasty
}

\author{
Jin $\mathrm{Li}^{1+}$, Ruikang Liư ${ }^{2+}$, Saroj Rai ${ }^{3}$, Renhao $\mathrm{Ze}^{1}$, Xin Tang ${ }^{1}$ and Pan Hong ${ }^{1 *}$ (D)
}

\begin{abstract}
Background: The optimal dosage and administration approach of tranexamic acid (TXA) in primary total knee arthroplasty (TKA) remains controversial. In light of recently published 14 randomized controlled trials (RCTs), the study aims to incorporate the newly found evidence and compare the efficacy and safety of intra-articular (IA) vs. intravenous (IV) application of TXA in primary TKA.

Methods: PubMed, Embase, Web of Science, and Cochrane Library were searched for RCTs comparing IA with IV TXA for primary TKA. Primary outcomes included total blood loss (TBL) and drain output. Secondary outcomes included hidden blood loss ( $\mathrm{HBL}$ ), hemoglobin $(\mathrm{Hb})$ fall, blood transfusion rate, perioperative complications, length of hospital stay, and tourniquet time.

Result: In all, 34 RCTs involving 3867 patients were included in our meta-analysis. Significant advantages of IA were shown on $\mathrm{TBL}(\mathrm{MD}=33.38,95 \% \mathrm{Cl}=19.24$ to $47.51, P<0.001)$, drain output $(\mathrm{MD}=28.44,95 \% \mathrm{Cl}=2.61$ to $54.27, P$ $=0.03)$, and postoperative day (POD) $3+\mathrm{Hb}$ fall $(\mathrm{MD}=0.24,95 \% \mathrm{Cl}=0.09$ to $0.39, P=0.001)$ compared with $\mathrm{IV}$. There existed no significant difference on HBL, POD1 and POD2 Hb fall, blood transfusion rate, perioperative complications, length of hospital stay, and tourniquet time between IA and IV.

Conclusion: Intra-articular administration of TXA is superior to intravenous in primary TKA patients regarding the performance on TBL, drain output, and POD3+ Hb fall, without increased risk of perioperative complications. Therefore, intra-articular administration is the recommended approach in clinical practice for primary TKA.
\end{abstract}

Keywords: Tranexamic acid, Total knee arthroplasty, Intra-articular administration, Intravenous administration

\footnotetext{
* Correspondence: hongpan2013@foxmail.com

${ }^{\dagger}$ Jin Li and Ruikang Liu contributed equally to this work and are the co-first authors.

'Department of Orthopaedic Surgery, Union Hospital, Tongji Medical College, Huazhong University of Science and Technology, Wuhan 430022, China Full list of author information is available at the end of the article
}

(c) The Author(s). 2020 Open Access This article is licensed under a Creative Commons Attribution 4.0 International License, which permits use, sharing, adaptation, distribution and reproduction in any medium or format, as long as you give appropriate credit to the original author(s) and the source, provide a link to the Creative Commons licence, and indicate if changes were made. The images or other third party material in this article are included in the article's Creative Commons licence, unless indicated otherwise in a credit line to the material. If material is not included in the article's Creative Commons licence and your intended use is not permitted by statutory regulation or exceeds the permitted use, you will need to obtain permission directly from the copyright holder. To view a copy of this licence, visit http://creativecommons.org/licenses/by/4.0/ The Creative Commons Public Domain Dedication waiver (http://creativecommons.org/publicdomain/zero/1.0/) applies to the data made available in this article, unless otherwise stated in a credit line to the data. 


\section{Background}

Total knee arthroplasty (TKA) is a common major orthopedic surgery, and the demand is still increasing due to human longevity and large population suffering from knee osteoarthritis (OA) around the world [1, 2].

TKA is an effective choice for end-stage OA [3]. But it is a major operation especially for the geriatric population, and the postoperative reduced hemoglobin $(\mathrm{Hb})$ might require blood transfusion and potentially result in delayed physical rehabilitation, longer hospital stay, and higher medical cost [4].

Tranexamic acid (TXA) has been widely used in many orthopedic surgeries for controlling blood loss [5]. Its safety and efficacy has been validated by many studies [6-8]. However, the optimal administration approach for primary TKA remains to be investigated. Oral administration and intravenous (IV) administration have been validated as an effective approach, but there are potential risks of thromboembolic complications [9, 10]. Besides, intra-articular (IA) administration provides a maximum concentration at the bleeding site with limited systemic influence [11].

Gianakos et al. [12] published the latest meta-analysis on IA vs. IV in 2018, and it demonstrated the superiority of IA over IV administration. However, with the publication of 14 new randomized controlled trial (RCT) results thereafter [13-26], it is imperative to perform a new meta-analysis to corroborate or repudiate the conclusion of Gianakos et al., which is the purpose of our study.

\section{Methods}

Our meta-analysis was conducted in accordance with the guidelines of the PRISMA (Preferred Reporting Items for Systematic Reviews and Meta-Analyses statement) [27]. We did not publish a protocol for this study.

\section{Literature search}

Four electronic databases including PubMed, Embase, Web of Science, and Cochrane Library were searched. Searching was conducted until April 20, 2020, with the following search terms: ("tranexamic acid" OR "TXA") AND ("total knee arthroplasty" OR "total knee replacement" OR “TKA" ). Literatures were limited to English publication. All studies were full text available. Unpublished investigations were not included.

\section{Selection criteria}

Two independent reviewers performed the search, removed duplicate records, reviewed the titles and abstracts, and identified studies as included, excluded, or uncertain. Full-text articles were reviewed to determine eligibility if identified uncertain. Disagreements were discussed with a third reviewer.
We retrieved all RCTs that compared IA with IV administration of TXA in patients receiving primary TKA. Inclusion criteria were (1) patients who underwent primary TKA, (2) comparative studies of IA vs. IV administration of TXA, (3) availability of full text, and (4) English publications. Exclusion criteria were (1) noncohort studies, (2) retrospective cohort studies, (3) reviews, and (4) unpublished studies.

\section{Data extraction}

The following data were extracted: characteristics of study (design, country, no. of patients, age, sex, body mass index, follow-up, and conclusion), method of administration and operation (IV or IA dosage, type of operation, and surgical approach), and surgical protocols (thromboprophylaxis, DVT screening, prosthetic properties, blood transfusion protocol, tourniquet application, and drainage).

Primary outcomes included total blood loss (TBL), which was calculated by the Gross formula or $\mathrm{Hb}$

Table 1 Modified Jadad scale

\begin{tabular}{|c|c|}
\hline Item assessed & Score \\
\hline \multicolumn{2}{|c|}{ Was the study described as randomized? } \\
\hline Yes & +1 \\
\hline No & 0 \\
\hline \multicolumn{2}{|c|}{ Was the method of randomization appropriate? } \\
\hline Yes & +1 \\
\hline No & -1 \\
\hline Not described & 0 \\
\hline \multicolumn{2}{|c|}{ Was the study described as blinded? } \\
\hline Yes & +1 \\
\hline No & 0 \\
\hline \multicolumn{2}{|c|}{ Was the method of blinding appropriate? } \\
\hline Yes & +1 \\
\hline No & -1 \\
\hline Not described & 0 \\
\hline \multicolumn{2}{|c|}{ Was there a description of withdrawals and dropouts? } \\
\hline Yes & +1 \\
\hline No & 0 \\
\hline \multicolumn{2}{|c|}{ Was there a clear description of the inclusion/exclusion criteria? } \\
\hline Yes & +1 \\
\hline No & 0 \\
\hline \multicolumn{2}{|c|}{ Was the method used to assess adverse effects described? } \\
\hline Yes & +1 \\
\hline No & 0 \\
\hline \multicolumn{2}{|c|}{ Was the method of statistical analysis described? } \\
\hline Yes & +1 \\
\hline No & 0 \\
\hline
\end{tabular}




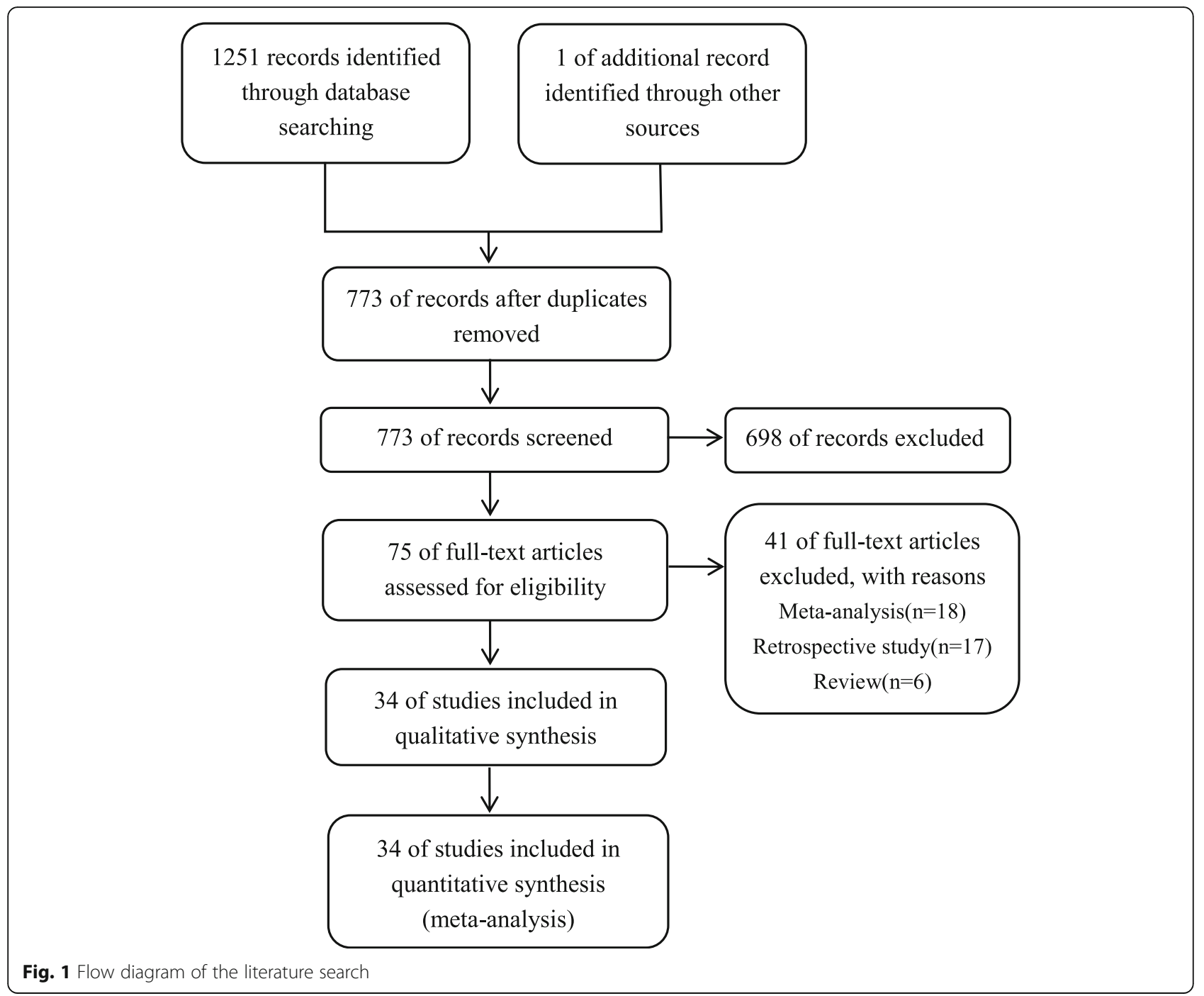

balance method [28, 29], and drain output. Secondary outcomes included hidden blood loss (HBL), $\mathrm{Hb}$ fall, blood transfusion rate, and perioperative complications including deep vein thrombosis (DVT), pulmonary embolism (PE), wound infection, and other vascular events. The duration of tourniquet application and length of hospital stay were also recorded and analyzed. Missing data were obtained from corresponding authors if possible.

\section{Quality assessment}

We assessed the qualities of included studies according to the criteria of the Cochrane Handbook for Systematic Reviews of Interventions [30]. The strength of evidence for each major outcome was evaluated according to the 8-point modified Jadad scale (Table 1) [31]. A study scoring above 4 was considered qualified. A study scoring above or equal to 7 was considered as high-quality evidence.

\section{Assessment of bias}

The risk of bias in individual studies was divided into five parts: selection bias (random generation sequence and allocation concealment), performance and detection bias (blind), attrition bias (incomplete data), reporting bias (selective reporting), and other biases. Publication bias across studies would be shown by funnel plot if necessary.

\section{Statistical analysis}

We analyzed continuous data by mean difference (MD) and its corresponding 95\% confidence interval (CI). Odds ratio (OR) and its corresponding 95\% CI were calculated for dichotomous data. We assessed heterogeneity by using the $I^{2}$ statistic. $I^{2}$ value above $50 \%$ was considered as high heterogeneity and a random-effects model would be used, while a value below $50 \%$ was considered as low heterogeneity and a fixed-effects model would be adopted [32]. Subgroup analyses would be considered 
Table 2 Characteristics of the study

\begin{tabular}{|c|c|c|c|c|c|c|c|c|c|c|c|c|}
\hline \multirow[t]{2}{*}{ Study } & \multirow[t]{2}{*}{ Design } & \multirow[t]{2}{*}{ Country } & \multicolumn{2}{|c|}{$\begin{array}{l}\text { No. of } \\
\text { patients }\end{array}$} & \multicolumn{2}{|c|}{ Age (years) } & \multicolumn{2}{|c|}{$\begin{array}{l}\text { Sex (male/ } \\
\text { female) }\end{array}$} & \multicolumn{2}{|c|}{ BMI $\left(\mathrm{kg} / \mathrm{m}^{2}\right)$} & \multirow[t]{2}{*}{$\begin{array}{l}\text { Follow- } \\
\text { up }\end{array}$} & \multirow[t]{2}{*}{ Conclusion } \\
\hline & & & IV & IA & IV & IA & IV & IA & IV & IA & & \\
\hline Jules-Elysee et al. [14] & $\mathrm{RCT}$ & USA & 31 & 32 & $65.6 \pm 8.4$ & $65.0 \pm 6.9$ & $11 / 20$ & $12 / 20$ & $31.6 \pm 7.1$ & $31.1 \pm 5.2$ & Unclear & $I V>I A$ \\
\hline $\begin{array}{l}\text { Laoruengthana et al. } \\
\text { [13] }\end{array}$ & $\mathrm{RCT}$ & Thailand & 76 & 75 & $\begin{array}{l}64.01 \pm \\
7.68\end{array}$ & $\begin{array}{l}64.81 \pm \\
8.06\end{array}$ & $62 / 14$ & $63 / 12$ & $27.8 \pm 5.2$ & $27.6 \pm 4.2$ & Unclear & $\mathrm{IA}>\mathrm{IV}$ \\
\hline Zhang et al. $[15,52]$ & $\mathrm{RCT}$ & China & 50 & 50 & $\begin{array}{l}63.12 \pm \\
8.79\end{array}$ & $\begin{array}{l}59.86 \pm \\
12.01\end{array}$ & $12 / 38$ & $10 / 40$ & $23.9 \pm 4.7$ & $25.0 \pm 4.3$ & $\begin{array}{l}6 \\
\text { months }\end{array}$ & $\mathrm{IA}>\mathrm{IV}$ \\
\hline Abdel et al. [20] & $\mathrm{RCT}$ & USA & 320 & 320 & 66 & 67 & $\begin{array}{l}127 / \\
193\end{array}$ & $\begin{array}{l}133 / \\
187\end{array}$ & 31.3 & 31.6 & Unclear & $I V>I A$ \\
\hline Ahmed et al. [23] & $\mathrm{RCT}$ & Pakistan & 70 & 70 & $\begin{array}{l}63.30 \pm \\
9.51\end{array}$ & $\begin{array}{l}64.39 \pm \\
9.07\end{array}$ & $28 / 42$ & $32 / 38$ & Unclear & Unclear & Unclear & $\mathrm{IA}>\mathrm{IV}$ \\
\hline $\begin{array}{l}\text { López-Hualda et al. } \\
\text { [21] }\end{array}$ & $\mathrm{RCT}$ & Spain & 30 & 30 & $73.1 \pm 7.3$ & $72.9 \pm 7.1$ & $6 / 24$ & $11 / 19$ & Unclear & Unclear & 1 year & $\mathrm{IA}>\mathrm{IV}$ \\
\hline George et al. [16] & $\mathrm{RCT}$ & India & 55 & 58 & 64.1 & 63.8 & $24 / 31$ & $14 / 44$ & 29.4 & 31.1 & 6 weeks & Neutral \\
\hline $\begin{array}{l}\text { Subramanyam et al. } \\
\text { [19] }\end{array}$ & $\mathrm{RCT}$ & India & 91 & 91 & $62.9 \pm 6.8$ & $62.7 \pm 7.5$ & $31 / 60$ & $35 / 56$ & 28.9 & 29.9 & 6 weeks & Neutral \\
\hline Wei et al. [18] & $\mathrm{RCT}$ & China & 32 & 32 & $\begin{array}{l}66.47 \pm \\
8.28\end{array}$ & $\begin{array}{l}66.43 \pm \\
7.69\end{array}$ & $14 / 18$ & $16 / 16$ & $32.4 \pm 3.7$ & $34.2 \pm 5.0$ & $\begin{array}{l}3 \\
\text { months }\end{array}$ & Neutral \\
\hline Goyal et al. [36] & $\mathrm{RCT}$ & Australia & 85 & 83 & $68.8 \pm 7.4$ & $66.7 \pm 8.9$ & $40 / 47$ & $38 / 43$ & $30.3 \pm 6.1$ & $31.0 \pm 5.3$ & 1 & Neutral \\
\hline Lacko et al. [22] & $\mathrm{RCT}$ & Slovakia & 30 & 30 & $68.4 \pm 7.2$ & $67.5 \pm 7.7$ & $12 / 18$ & $13 / 17$ & $31.1 \pm 4.7$ & $31.9 \pm 4.7$ & $\begin{array}{l}3 \\
\text { months }\end{array}$ & $I V>I A$ \\
\hline Maniar et al. [33] & $\mathrm{RCT}$ & India & 50 & 25 & $65.7 \pm 7.6$ & $62.2 \pm 7.1$ & $7 / 43$ & $2 / 23$ & $30.2 \pm 4.5$ & $30.3 \pm 3.9$ & $\begin{array}{l}3 \\
\text { months }\end{array}$ & Neutral \\
\hline Prakash et al. [26] & $\mathrm{RCT}$ & India & 50 & 50 & 70.2 & 71 & NR & NR & Unclear & Unclear & $\begin{array}{l}3 \\
\text { months }\end{array}$ & $I V>I A$ \\
\hline Song et al. [35] & $\mathrm{RCT}$ & $\begin{array}{l}\text { South } \\
\text { Korea }\end{array}$ & 50 & 50 & $69.2 \pm 6.4$ & $69.8 \pm 6.8$ & $6 / 44$ & $8 / 42$ & $\begin{array}{l}26.52 \pm \\
3.3\end{array}$ & $\begin{array}{l}26.96 \pm \\
4.2\end{array}$ & $\begin{array}{l}3 \\
\text { months }\end{array}$ & Neutral \\
\hline Stowers et al. [24] & $\mathrm{RCT}$ & $\begin{array}{l}\text { New } \\
\text { Zealand }\end{array}$ & 51 & 60 & $71 \pm 8.6$ & $70 \pm 8.5$ & $27 / 24$ & $28 / 32$ & $31.2 \pm 5.5$ & $31.2 \pm 5.5$ & $\begin{array}{l}6 \\
\text { months }\end{array}$ & Neutral \\
\hline Uğurlu et al. [34] & $\mathrm{RCT}$ & USA & 40 & 42 & $69.4 \pm 7.5$ & $70.6 \pm 8.6$ & $11 / 29$ & $9 / 33$ & $30.8 \pm 5.3$ & $31.1 \pm 5.4$ & 10 days & Neutral \\
\hline Wang et al. [11, 23] & $\mathrm{RCT}$ & China & 50 & 50 & $\begin{array}{l}67.42 \pm \\
8.20\end{array}$ & $\begin{array}{l}67.98 \pm \\
5.97\end{array}$ & $14 / 36$ & $14 / 36$ & $26.7 \pm 3.4$ & $25.9 \pm 3.8$ & 5 weeks & $\mathrm{IA}>\mathrm{IV}$ \\
\hline Zekcer et al. [25] & $\mathrm{RCT}$ & Brazil & 30 & 30 & 65.7 & 65.7 & $6 / 24$ & $9 / 21$ & Unclear & Unclear & Unclear & Neutral \\
\hline Aggarwal et al. [39] & $\mathrm{RCT}$ & India & 35 & 35 & $\begin{array}{l}58.77 \pm \\
10.14\end{array}$ & $\begin{array}{l}55.66 \pm \\
8.71\end{array}$ & $13 / 22$ & $12 / 23$ & $\begin{array}{l}26.33 \pm \\
3.79\end{array}$ & $\begin{array}{l}27.33 \pm \\
4.63\end{array}$ & $\begin{array}{l}6 \\
\text { months }\end{array}$ & $\mathrm{IA}>\mathrm{IV}$ \\
\hline Chen et al. $[29,53]$ & $\mathrm{RCT}$ & Singapore & 50 & 50 & $65 \pm 8$ & $65 \pm 8$ & $15 / 35$ & $10 / 40$ & $28 \pm 5$ & $28 \pm 7$ & 1 & Neutral \\
\hline Drosos et al. [38] & $\mathrm{RCT}$ & Greece & 30 & 30 & $\begin{array}{l}69.27 \pm \\
7.21\end{array}$ & $\begin{array}{l}71.10 \pm \\
6.32\end{array}$ & $6 / 24$ & $6 / 24$ & $\begin{array}{l}32.79 \pm \\
5.04\end{array}$ & $\begin{array}{l}33.38 \pm \\
6.08\end{array}$ & 1 & Neutral \\
\hline Keyhani et al. [42] & $\mathrm{RCT}$ & Iran & 40 & 40 & $68.4 \pm 10.4$ & $67 \pm 11.9$ & $26 / 14$ & $23 / 17$ & $32.7 \pm 5.5$ & $31.3 \pm 5.4$ & 2 weeks & Neutral \\
\hline May et al. [37] & $\mathrm{RCT}$ & USA & 69 & 62 & $65.0 \pm 9.6$ & $63.0 \pm 10.6$ & $11 / 58$ & $18 / 44$ & 33.8 & 33.8 & $\begin{array}{l}1 \\
\text { month }\end{array}$ & Neutral \\
\hline Pinsornsak et al. [41] & $\mathrm{RCT}$ & Thailand & 30 & 30 & $\begin{array}{l}69.97 \pm \\
7.55\end{array}$ & $\begin{array}{l}67.63 \pm \\
7.96\end{array}$ & $7 / 23$ & $5 / 25$ & $\begin{array}{l}26.52 \pm \\
3.7\end{array}$ & $\begin{array}{l}27.96 \pm \\
4.99\end{array}$ & 2 weeks & Neutral \\
\hline Tzatzairis et al. [40] & $\mathrm{RCT}$ & Greece & 40 & 40 & $\begin{array}{l}69.55 \pm \\
6.61\end{array}$ & $\begin{array}{l}69.10 \pm \\
8.68\end{array}$ & $9 / 31$ & $7 / 33$ & $\begin{array}{l}32.60 \pm \\
4.09\end{array}$ & $\begin{array}{l}32.60 \pm \\
4.50\end{array}$ & 6 weeks & Neutral \\
\hline Aguilera et al. [43] & $\mathrm{RCT}$ & Spain & 50 & 50 & $\begin{array}{l}72.49 \pm \\
7.68\end{array}$ & $\begin{array}{l}72.53 \pm \\
6.60\end{array}$ & $38 / 12$ & $32 / 18$ & $\begin{array}{l}30.20 \pm \\
4.10\end{array}$ & $\begin{array}{l}30.89 \pm \\
4.37\end{array}$ & $\begin{array}{l}2 \\
\text { months }\end{array}$ & Neutral \\
\hline Digas et al. [44] & $\mathrm{RCT}$ & Greece & 30 & 30 & $70 \pm 6.5$ & $71 \pm 7.0$ & $2 / 28$ & $7 / 23$ & Unclear & Unclear & 1 year & $\mathrm{IA}>\mathrm{IV}$ \\
\hline Öztaş et al. [45] & $\mathrm{RCT}$ & Turkey & 30 & 30 & 68.56 & 67.06 & $5 / 25$ & $4 / 26$ & Unclear & Unclear & $\begin{array}{l}3 \\
\text { months }\end{array}$ & $I A>I V$ \\
\hline
\end{tabular}


Table 2 Characteristics of the study (Continued)

\begin{tabular}{|c|c|c|c|c|c|c|c|c|c|c|c|c|}
\hline \multirow[t]{2}{*}{ Study } & \multirow[t]{2}{*}{ Design } & \multirow[t]{2}{*}{ Country } & \multicolumn{2}{|c|}{$\begin{array}{l}\text { No. of } \\
\text { patients }\end{array}$} & \multicolumn{2}{|l|}{ Age (years) } & \multicolumn{2}{|c|}{$\begin{array}{l}\text { Sex (male/ } \\
\text { female) }\end{array}$} & \multicolumn{2}{|c|}{ BMI $\left(\mathrm{kg} / \mathrm{m}^{2}\right)$} & \multirow[t]{2}{*}{$\begin{array}{l}\text { Follow- } \\
\text { up }\end{array}$} & \multirow[t]{2}{*}{ Conclusion } \\
\hline & & & IV & IA & IV & IA & IV & IA & IV & IA & & \\
\hline $\begin{array}{l}\text { Gomez-Barrena et al. } \\
\text { [46] }\end{array}$ & $\mathrm{RCT}$ & Spain & 39 & 39 & $71.8 \pm 10.3$ & $70.1 \pm 9.1$ & $25 / 14$ & $26 / 13$ & $30.2 \pm 4.2$ & $30.4 \pm 4.1$ & $\begin{array}{l}1 \\
\text { month }\end{array}$ & Neutral \\
\hline Patel et al. [47] & $\mathrm{RCT}$ & USA & 42 & 47 & $64.9 \pm 7.8$ & $64.8 \pm 9.7$ & $10 / 32$ & $13 / 34$ & $35.8 \pm 8.6$ & $32.7 \pm 7.0$ & 2 weeks & Neutral \\
\hline Sarzaeem et al. [48] & $\mathrm{RCT}$ & Iran & 50 & 100 & $66.9 \pm 7.2$ & $67.8 \pm 7.2$ & $7 / 43$ & $13 / 87$ & $31.6 \pm 2.7$ & $31.5 \pm 3.4$ & Unclear & Neutral \\
\hline Soni et al. [49] & $\mathrm{RCT}$ & India & 40 & 40 & $\begin{array}{l}69.05 \pm \\
4.10\end{array}$ & $\begin{array}{l}69.45 \pm \\
4.71\end{array}$ & $19 / 21$ & $17 / 23$ & Unclear & Unclear & 6 weeks & Neutral \\
\hline Seo et al. [50] & $\mathrm{RCT}$ & $\begin{array}{l}\text { South } \\
\text { Korea }\end{array}$ & 50 & 50 & $66.8 \pm 6.3$ & $67.5 \pm 6.6$ & $6 / 44$ & $5 / 45$ & $28.1 \pm 3.1$ & $27.8 \pm 3.5$ & $\begin{array}{l}2 \\
\text { months }\end{array}$ & $\mathrm{IA}>\mathrm{IV}$ \\
\hline Maniar et al. [51] & $\mathrm{RCT}$ & India & 160 & 40 & $67.4 \pm 8.1$ & $67.4 \pm 7.9$ & $36 / 124$ & $6 / 34$ & $29.2 \pm 5.4$ & $30.9 \pm 5.2$ & $\begin{array}{l}3 \\
\text { months }\end{array}$ & Neutral \\
\hline
\end{tabular}

$R C T$ randomized controlled trial, $I V$ intravenous group, $I A$ intra-articular group, TKA total knee arthroplasty, BMI body mass index

when meeting high heterogeneity. Statistical analyses were performed using Review Manager 5.3 software. Forrest plots were used to describe the primary results of the meta-analysis. Funnel plots for primary outcomes (TBL and drain output) were generated to evaluate the potential publication bias. $P$ value $<0.05$ was considered statistically significant.

Formal ethical approval was deemed not necessary in our meta-analysis.

\section{Result}

\section{Search results}

Figure 1 shows detailed steps of the literature search, in which 773 studies were reviewed: 698 studies were excluded after screening titles and abstracts, and the remaining 75 studies were reviewed in full text. After excluding 41 studies according to selection criteria, 34 studies encompassing 3867 patients were included in our study [13-26, 29, 33-51].

\section{Study characteristics and quality assessments}

As shown in Table 2, the sample size of the included studies ranged from 25 to 320 , and the mean age of patients ranged from 57 to 73 . Nine of the studies (9/34, 26.5\%) favored IA administration, while four of the studies $(4 / 34,11.8 \%)$ preferred IV administration.

Methods of administration and types of operation are presented in Table 3. One study (Maniar et al.) included four IV groups and another study (Maniar et al.) included two IV groups [33, 51]. One study (Sarzaeem et al.) had two IA groups with different dosages [48]. Unilateral TKA was performed in 31 studies (31/34, 91.2\%) while bilateral TKA was performed in three studies (31/34, 8.8\%). Twenty-four studies (24/34, 70.6\%) adopted medial parapatellar, four $(4 / 34,11.8 \%)$ chose the midvastus approach, and two studies $(2 / 34,5.9 \%)$ used subvastus parapatellar, while the approach was unclear in the rest six studies $(6 / 34,17.6 \%)$.
Table 4 summarizes the detailed surgical protocols. Low-molecular-weight heparin (LMWH) was the preferred prophylactic choice for thrombosis $(21 / 34,61.8 \%)$, following by pumping exercise and compression stocking $(7 / 34,20.6 \%)$, and aspirin $(6 / 34,17.6 \%)$. Both Doppler ultrasound and clinical examination were the most commonly used screening method for DVT (16/34, $47.1 \%)$, and chest CT was used in five studies (5/34, $14.7 \%)$, while nine $(9 / 34,26.5 \%)$ remained unclear. Cemented prosthesis was adopted in 27 studies $(27 / 34$, $79.4 \%)$, tourniquet was used in 31 studies $(31 / 34,91.2 \%)$, and 19 of the studies $(31 / 34,55.9 \%)$ clamped the drain tube after the operation.

Quality assessment and assessment of bias are presented in Table 5. In all, 25 studies (25/34, 73.5\%) are high-quality and nine $(9 / 34,26.5 \%)$ are moderate-quality evidences.

\section{Meta-analysis of outcomes}

All the results are listed in Table 6, including primary outcomes, secondary outcomes, three subgroup analyses, and three low heterogeneity analyses.

\section{Total blood loss}

Eighteen studies provided valid data of TBL on $1656 \mathrm{pa}-$ tients. Given the presence of significant heterogeneity among studies $\left(P<0.001, I^{2}=81 \%\right)$, we used a randomeffects model for analysis. IA administration showed a significant advantage compared to IV administration ( $\mathrm{MD}=$ 63.99, $95 \% \mathrm{CI}=27.81$ to $100.16, P<0.001)$. Concerning about the high heterogeneity, we performed a sensitivity analysis based on the risk of bias and got another lower heterogeneity result (Fig. 2$)$ by analyzing 13 studies $(P=$ $0.34, I^{2}=11 \%$ ) with a fixed-effects model, which still revealed a significant superiority of IA administration (MD $=33.38,95 \% \mathrm{CI}=19.24$ to $47.51, P<0.001)$. Publication bias is shown by a funnel plot (Fig. 3 ). 
Table 3 Methods of administration and operation

\begin{tabular}{|c|c|c|c|c|}
\hline Study & IV dosage & IA dosage & Type of operation & Surgical approach \\
\hline $\begin{array}{l}\text { Jules-Elysee et al. } \\
\text { [14] }\end{array}$ & $1 \mathrm{~g}$ TXA $\times$ two doses; POPO & $\begin{array}{l}3 \mathrm{~g} \text { TXA } \times \text { one dose; before } \\
\text { tourniquet release }\end{array}$ & Primary unilateral TKA & Unclear \\
\hline $\begin{array}{l}\text { Laoruengthana } \\
\text { et al. [13] }\end{array}$ & 10 mg/kg TXA $\times$ one dose; 10 & $\begin{array}{l}15 \mathrm{mg} / \mathrm{kg} \text { TXA } \times \text { one dose; before } \\
\text { closure }\end{array}$ & Primary unilateral TKA & Medial parapatellar \\
\hline $\begin{array}{l}\text { Zhang et al. [15, } \\
\text { 52] }\end{array}$ & $\begin{array}{l}20 \mathrm{mg} / \mathrm{kg} \text { TXA } \times \text { one dose; } \\
\text { PEO }\end{array}$ & $3 \mathrm{~g}$ TXA $\times$ one dose; after closure & Primary unilateral TKA & Medial parapatellar \\
\hline Abdel et al. [20] & $1 \mathrm{~g}$ TXA $\times$ one dose; PEO & $\begin{array}{l}3 \mathrm{~g} \text { TXA } \times \text { one dose; after } \\
\text { cemented }\end{array}$ & Primary unilateral TKA & $\begin{array}{l}\text { Medial parapatellar or } \\
\text { midvastus approach }\end{array}$ \\
\hline Ahmed et al. [17] & $1.5 \mathrm{~g}$ TXA $\times$ one dose; PTO & $1.5 \mathrm{~g}$ TXA $\times$ one dose; while closure & $\begin{array}{l}\text { Primary simultaneous } \\
\text { bilateral TKA }\end{array}$ & Unclear \\
\hline $\begin{array}{l}\text { López-Hualda } \\
\text { et al. [21] }\end{array}$ & $1 \mathrm{~g}$ TXA $\times$ one dose; PEO & 1 g TXA $\times$ one dose; after closure & Primary unilateral TKA & Medial parapatellar \\
\hline George et al. [16] & $\begin{array}{l}10 \mathrm{mg} / \mathrm{kg} \text { TXA } \times \text { two doses; } \\
\text { POPO }\end{array}$ & $\begin{array}{l}1.5 \mathrm{~g} \text { TXA } \times \text { one dose; before } \\
\text { closure }\end{array}$ & Primary unilateral TKA & Medial parapatellar \\
\hline $\begin{array}{l}\text { Subramanyam } \\
\text { et al. [19] }\end{array}$ & $\begin{array}{l}10 \mathrm{mg} / \mathrm{kg} \text { TXA } \times \text { one dose; } \\
\text { PEO }\end{array}$ & $1.5 \mathrm{~g}$ TXA $\times$ one dose; after closure & Primary unilateral TKA & Medial parapatellar \\
\hline Wei et al. [18] & $\begin{array}{l}10 \mathrm{mg} / \mathrm{kg} \text { TXA } \times \text { one dose; } \\
\text { PEO }\end{array}$ & $\begin{array}{l}1 \mathrm{~g} \text { TXA } \times \text { one dose; before } \\
\text { tourniquet release }\end{array}$ & Primary unilateral TKA & Unclear \\
\hline Goyal et al. [36] & $\begin{array}{l}1 \mathrm{~g} \text { TXA } \times \text { three doses; IO/ } \\
\text { PTO/PTO }\end{array}$ & $3 \mathrm{~g}$ TXA $\times$ one dose; after closure & Primary unilateral TKA & Medial parapatellar \\
\hline Lacko et al. [22] & $\begin{array}{l}10 \mathrm{mg} / \mathrm{kg} \text { TXA } \times \text { two doses; } \\
\text { POPO }\end{array}$ & $\begin{array}{l}3 \mathrm{~g} \text { TXA } \times \text { one dose; after } \\
\text { cemented }\end{array}$ & Primary unilateral TKA & Medial parapatellar \\
\hline \multirow[t]{2}{*}{ Maniar et al. [33] } & $\begin{array}{l}10 \mathrm{mg} / \mathrm{kg} \text { TXA } \times \text { two doses } \\
\text { (bilateral); IO }\end{array}$ & $\begin{array}{l}3 \mathrm{~g} \text { TXA } \times \text { two doses(bilateral); after } \\
\text { cemented }\end{array}$ & $\begin{array}{l}\text { Primary simultaneous } \\
\text { bilateral TKA }\end{array}$ & Midvastus approach \\
\hline & $\begin{array}{l}10 \mathrm{mg} / \mathrm{kg} \text { TXA } \times \text { three doses; } \\
\text { POIOPO }\end{array}$ & & & \\
\hline Prakash et al. [26] & $\begin{array}{l}10 \mathrm{mg} / \mathrm{kg} \text { TXA } \times \text { three doses; } \\
\text { POIOPO }\end{array}$ & $3 \mathrm{~g}$ TXA $\times$ one dose; before closure & Primary unilateral TKA & Medial parapatellar \\
\hline Song et al. [35] & $\begin{array}{l}10 \mathrm{mg} / \mathrm{kg} \text { TXA } \times \text { three doses; } \\
\text { POIOPO }\end{array}$ & $1.5 \mathrm{~g}$ TXA $\times$ one dose; after closure & Primary bilateral TKA & Medial parapatellar \\
\hline Stowers et al. [24] & $1 \mathrm{~g}$ TXA $\times$ one dose; 10 & $1 \mathrm{~g}$ TXA $\times$ one dose; after closure & Primary unilateral TKA & Medial parapatellar \\
\hline Uğurlu et al. [34] & $\begin{array}{l}20 \mathrm{mg} / \mathrm{kg} \text { TXA } \times \text { one dose; } \\
\text { PEO }\end{array}$ & $3 \mathrm{~g}$ TXA $\times$ one dose; after closure & Primary unilateral TKA & Medial parapatellar \\
\hline $\begin{array}{l}\text { Wang et al. [11, } \\
\text { 23] }\end{array}$ & $1 \mathrm{~g}$ TXA $\times$ one dose; $1 O$ & 1 g TXA $\times$ one dose; before closure & Primary unilateral TKA & Medial parapatellar \\
\hline Zekcer et al. [25] & $\begin{array}{l}20 \mathrm{mg} / \mathrm{kg} \text { TXA } \times \text { one dose; } \\
\text { unclear }\end{array}$ & $\begin{array}{l}1.5 \mathrm{~g} \text { TXA } \times \text { one dose; before } \\
\text { tourniquet release }\end{array}$ & Primary unilateral TKA & Unclear \\
\hline $\begin{array}{l}\text { Aggarwal et al. } \\
\text { [39] }\end{array}$ & $\begin{array}{l}15 \mathrm{mg} / \mathrm{kg} \text { TXA } \times \text { two dose; } \\
\text { IOPO }\end{array}$ & $\begin{array}{l}15 \mathrm{mg} / \mathrm{kg} \text { TXA } \times \text { one dose; before } \\
\text { closure }\end{array}$ & $\begin{array}{l}\text { Primary simultaneous } \\
\text { bilateral TKA }\end{array}$ & Medial parapatellar \\
\hline Chen et al. $[29,53]$ & $1.5 \mathrm{~g}$ TXA $\times$ one dose; 10 & $\begin{array}{l}1.5 \mathrm{~g} \text { TXA } \times \text { one dose; after } \\
\text { cemented }\end{array}$ & Primary unilateral TKA & Medial parapatellar \\
\hline Drosos et al. [38] & $1 \mathrm{~g}$ TXA $\times$ one dose; PEO & $1 \mathrm{~g}$ TXA $\times$ one dose; before closure & Primary unilateral TKA & Medial parapatellar \\
\hline Keyhani et al. [42] & $0.5 \mathrm{~g}$ TXA $\times$ one dose; 10 & $\begin{array}{l}1.5 \mathrm{~g} \text { TXA } \times \text { two doses; before/after } \\
\text { closure }\end{array}$ & Primary unilateral TKA & Medial parapatellar \\
\hline May et al. [37] & $1 \mathrm{~g}$ TXA $\times$ two doses; POPO & $2 \mathrm{~g}$ TXA $\times$ one dose; after closure & Primary unilateral TKA & Unclear \\
\hline $\begin{array}{l}\text { Pinsornsak et al. } \\
\text { [37] }\end{array}$ & $0.75 \mathrm{mg}$ TXA $\times$ one dose; $1 \mathrm{O}$ & $\begin{array}{l}0.75 \mathrm{mg} \times \text { one dose; before } \\
\text { tourniquet release }\end{array}$ & Primary unilateral TKA & Medial parapatellar \\
\hline Tzatzairis et al. [40] & $1 \mathrm{~g}$ TXA $\times$ one dose; PEO & 1 g TXA $\times$ one dose; after closure & Primary unilateral TKA & Medial parapatellar \\
\hline Aguilera et al. [43] & $1 \mathrm{~g}$ TXA $\times$ two doses; POIO & $\begin{array}{l}1 \mathrm{~g} \text { TXA } \times \text { one dose; after } \\
\text { cemented }\end{array}$ & Primary unilateral TKA & Medial parapatellar \\
\hline Digas et al. [44] & 15 mg/kg TXA $\times$ one dose; IO & $2 \mathrm{~g}$ TXA $\times$ one dose; after closure & Primary unilateral TKA & Medial parapatellar \\
\hline Öztaş et al. [45] & $\begin{array}{l}15 \mathrm{mg} / \mathrm{kg} \text { TXA } \times \text { two doses; } \\
\text { POPO }\end{array}$ & $\begin{array}{l}2 \mathrm{~g} \text { TXA } \times \text { one dose; before } \\
\text { tourniquet release }\end{array}$ & Primary unilateral TKA & Unclear \\
\hline
\end{tabular}


Table 3 Methods of administration and operation (Continued)

\begin{tabular}{|c|c|c|c|c|}
\hline Study & IV dosage & IA dosage & Type of operation & Surgical approach \\
\hline & $\begin{array}{l}10 \mathrm{mg} / \mathrm{kg} \text { TXA } \times \text { one dose; } 1- \\
\mathrm{h} \text { infusion }\end{array}$ & & & \\
\hline $\begin{array}{l}\text { Gomez-Barrena } \\
\text { et al. [46] }\end{array}$ & $\begin{array}{l}15 \mathrm{mg} / \mathrm{kg} \text { TXA } \times \text { two doses; } \\
\text { IOPO }\end{array}$ & $\begin{array}{l}3 \mathrm{~g} \text { TXA } \times \text { one dose; before }+ \text { after } \\
\text { closure }\end{array}$ & Primary unilateral TKA & Medial parapatellar \\
\hline Patel et al. [47] & 10 mg/kg TXA $\times$ one dose; 10 & $\begin{array}{l}2 \mathrm{~g} \text { TXA } \times \text { one dose; before } \\
\text { tourniquet release }\end{array}$ & Primary unilateral TKA & $\begin{array}{l}\text { Medial or subvastus } \\
\text { parapatellar }\end{array}$ \\
\hline \multirow{2}{*}{$\begin{array}{l}\text { Sarzaeem et al. } \\
\text { [48] }\end{array}$} & \multirow[t]{2}{*}{$1.5 \mathrm{~g}$ TXA $\times$ one dose; PTO } & $1.5 \mathrm{~g}$ TXA $\times$ one dose; after closure & \multirow[t]{2}{*}{ Primary unilateral TKA } & \multirow[t]{2}{*}{ Subvastus approach } \\
\hline & & $3 \mathrm{~g}$ TXA $\times$ one dose; before closure & & \\
\hline Soni et al. [49] & $\begin{array}{l}10 \mathrm{mg} / \mathrm{kg} \text { TXA } \times \text { three doses; } \\
\text { POIOPO }\end{array}$ & $\begin{array}{l}3 \mathrm{~g} \text { TXA } \times \text { one dose; before } \\
\text { tourniquet release }\end{array}$ & Primary unilateral TKA & Midvastus approach \\
\hline Seo et al. [50] & $1.5 \mathrm{~g}$ TXA $\times$ one dose; PTO & $1.5 \mathrm{~g}$ TXA $\times$ one dose; while closure & Primary unilateral TKA & Medial parapatellar \\
\hline \multirow[t]{4}{*}{ Maniar et al. [51] } & $10 \mathrm{mg} / \mathrm{kg}$ TXA $\times$ one dose; 10 & \multirow{4}{*}{$\begin{array}{l}3 \mathrm{~g} \text { TXA } \times \text { one dose; before } \\
\text { tourniquet release }\end{array}$} & \multirow[t]{4}{*}{ Primary unilateral TKA } & \multirow[t]{4}{*}{ Midvastus approach } \\
\hline & $\begin{array}{l}10 \mathrm{mg} / \mathrm{kg} \text { TXA } \times \text { two doses; } \\
\text { IOPO }\end{array}$ & & & \\
\hline & $\begin{array}{l}10 \text { mg/kg TXA } \times \text { two doses; } \\
\mathrm{POlO}\end{array}$ & & & \\
\hline & $\begin{array}{l}10 \mathrm{mg} / \mathrm{kg} \text { TXA } \times \text { three doses; } \\
\text { POIOPO }\end{array}$ & & & \\
\hline
\end{tabular}

$I O$ intraoperative dose, $I O P O$ intra- and postoperative doses, $P E O$ preoperative dose, $P O I O$ pre- and intraoperative doses, $P O I O P O$ all three doses, $P O P O$ pre- and postoperative doses, PTO postoperative dose, TXA tranexamic acid

\section{Drain output}

Seventeen studies involving 1494 patients provided valid data of drain output. Due to significant heterogeneity among studies $\left(P<0.001, I^{2}=93 \%\right)$, we used a randomeffects model for analysis. IA administration showed a significant advantage (Fig. 4) compared to IV administration $(\mathrm{MD}=28.44,95 \% \mathrm{CI}=2.61$ to $54.27, P=0.03)$.

Drainage volume was analyzed in subgroup based on the duration of tube clamping. For studies in which the drainage tube was clamped postoperatively less than two hours, a significant superiority was shown in the IA group compared to the IV group $(\mathrm{MD}=51.47,95 \% \mathrm{CI}=$ 6.02 to $96.92, P=0.03)$. Considering the high heterogeneity $\left(P<0.001, I^{2}=92 \%\right)$, a random-effects was used for analysis. There was no significant difference $(\mathrm{MD}=$ $12.40,95 \% \mathrm{CI}=-24.85$ to $49.65, P=0.51$ ) for studies in which the drainage tube was clamped postoperatively over $2 \mathrm{~h}$ with high heterogeneity $\left(P<0.001, I^{2}=89 \%\right)$.

\section{Hidden blood loss}

Only six studies including 640 patients reported HBL. Since there existed significant heterogeneity among studies $\left(P=0.006, I^{2}=69 \%\right)$, we used a random-effects model for analysis. There existed no significant difference between the IV and IA groups (MD $=7.57,95 \% \mathrm{CI}$ $=-60.34$ to $75.47, P=0.83)$ on HBL.

\section{Hemoglobin fall}

In all, 19 studies involving 1749 patients reported the data of postoperative $\mathrm{Hb}$ fall. Because different studies reported $\mathrm{Hb}$ of postoperative day (POD) 1 to 5 with high heterogeneity $\left(P<0.001, I^{2}=87 \%\right)$, we conducted subgroup analyses based on POD1, POD2, or POD3+.

Ten studies involving 1052 patients reported the POD1 Hb fall. The random-effects model $\left(P<0.001, I^{2}\right.$ $=91 \%)$ was used for analysis, and there was no significant difference between the IV and IA groups (MD $=-0.34$, $95 \% \mathrm{CI}=-0.70$ to $0.02, P=0.07)$. Regarding the high heterogeneity, a sensitivity analysis was performed and two studies were excluded $[14,48]$, then we got a lower heterogeneity result $\left(P=0.14, I^{2}=36 \%\right)$ by analyzing the rest of 8 studies including 839 patients with a fixed-effects model. No significant difference was shown between the IV and IA groups $(\mathrm{MD}=-0.01,95 \% \mathrm{CI}=-0.11$ to $0.13, P=0.86)$.

Eight studies involving 701 patients reported the POD2 $\mathrm{Hb}$ fall. Considering the significant heterogeneity among studies $\left(P<0.001, I^{2}=82 \%\right)$, we used a randomeffects model for analysis. There existed no significant difference between the IV and IA groups $(\mathrm{MD}=0.17$, $95 \% \mathrm{CI}=-0.20$ to $0.53, P=0.37$ ). We also performed a sensitivity analysis based on the risk of bias and excluded two studies $[23,39]$ and got a lower heterogeneity $(P=$ $0.11, I^{2}=44 \%$ ) result by analyzing the rest of six studies involving 531 patients with a fixed-effects model. No significant difference was shown between the IV and IA groups ( $\mathrm{MD}=-0.08,95 \% \mathrm{CI}=-0.25$ to $0.09, P=0.36$ ).

Six studies involving 637 patients reported the POD3+ $\mathrm{Hb}$ fall. Because of low heterogeneity among studies $(P$ $=0.18, I^{2}=34 \%$ ), a fixed-effects model was used for analysis. The IA group showed a significant advantage compared to the IV group $(\mathrm{MD}=0.24,95 \% \mathrm{CI}=0.09$ to $0.39, P=0.001)$. 
Table 4 Surgical protocols

\begin{tabular}{|c|c|c|c|c|c|c|}
\hline Study & Thromboprophylaxis & $\begin{array}{l}\text { DVT screening } \\
\text { method }\end{array}$ & $\begin{array}{l}\text { Prosthetic } \\
\text { properties }\end{array}$ & $\begin{array}{l}\text { Blood transfusion } \\
\text { protocol }\end{array}$ & Tourniquet & Drainage \\
\hline $\begin{array}{l}\text { Jules-Elysee et al. } \\
{[14]}\end{array}$ & Unclear & Unclear & Cemented & Unclear & Yes & $\begin{array}{l}\text { Clamped for } 4 \\
\text { h }\end{array}$ \\
\hline $\begin{array}{l}\text { Laoruengthana } \\
\text { et al. [13] }\end{array}$ & LMWH/warfarin & Unclear & Cemented & $\mathrm{Hb}<9.0 \mathrm{~g} / \mathrm{L}$ & Yes & $\begin{array}{l}\text { Clamped for } 3 \\
\mathrm{~h}\end{array}$ \\
\hline $\begin{array}{l}\text { Zhang et al. [15, } \\
\text { 52] }\end{array}$ & Rivaroxaban & Doppler ultrasound & Cemented & Unclear & Yes & Unclear \\
\hline Abdel et al. [20] & Aspirin/warfarin & Unclear & Cemented & $\begin{array}{l}\mathrm{Hb}<7.0 \mathrm{~g} / \mathrm{dL} \\
\mathrm{Hb}<10.0 \mathrm{~g} / \mathrm{dL}+ \\
\text { symptoms }\end{array}$ & Yes & Unclear \\
\hline Ahmed et al. [17] & Unclear & Unclear & Unclear & Unclear & Unclear & Unclear \\
\hline $\begin{array}{l}\text { López-Hualda et al. } \\
\text { [21] }\end{array}$ & Unclear & Unclear & Cemented & $\begin{array}{l}\mathrm{Hb}<8.0 \mathrm{~g} / \mathrm{dL}+ \\
\text { symptoms }\end{array}$ & Yes & Unclear \\
\hline George et al. [16] & LMWH/aspirin & Doppler ultrasound & Cemented & $\mathrm{Hb}<7.0 \mathrm{~g} / \mathrm{dL}$ & Yes & Unclear \\
\hline $\begin{array}{l}\text { Subramanyam } \\
\text { et al. [19] }\end{array}$ & $\begin{array}{l}\text { Aspirin } \\
\text { Calf pump }\end{array}$ & $\begin{array}{l}\text { Clinical examination } \\
\text { Doppler ultrasound }\end{array}$ & Cemented & $\begin{array}{l}\mathrm{Hb}<8.0 \mathrm{~g} / \mathrm{dL} \\
\mathrm{Hb}<10.0 \mathrm{~g} / \mathrm{dL}+ \\
\text { symptoms }\end{array}$ & Yes & No drain \\
\hline Wei et al. [18] & LMWH & Unclear & Cemented & $\begin{array}{l}\mathrm{Hb}<8.0 \mathrm{~g} / \mathrm{dL} \\
\mathrm{Hb}<10.0 \mathrm{~g} / \mathrm{dL}+ \\
\text { symptoms }\end{array}$ & Yes & Unclear \\
\hline Goyal et al. [36] & $\begin{array}{l}\text { LMWH/aspirin Compression } \\
\text { stocking }\end{array}$ & Doppler ultrasound & Hybrid & $\begin{array}{l}\mathrm{Hb}<7.0 \mathrm{~g} / \mathrm{dL} \\
\mathrm{Hb}<10.0 \mathrm{~g} / \mathrm{dL}+ \\
\text { symptoms }\end{array}$ & No & Closed \\
\hline Lacko et al. [22] & Unclear & Doppler ultrasound & Cemented & $\begin{array}{l}\mathrm{Hb}<8.0 \mathrm{~g} / \mathrm{dL} \\
\mathrm{Hb}<9.0+\text { symptoms }\end{array}$ & Yes & Unclear \\
\hline Maniar et al. [33] & $\begin{array}{l}\text { LMWH } \\
\text { Ankle pumping exercise } \\
\text { Compression stocking }\end{array}$ & $\begin{array}{l}\text { Clinical examination } \\
\text { Doppler ultrasound }\end{array}$ & Cemented & $\begin{array}{l}\mathrm{Hn}<8.5 \mathrm{~g} / \mathrm{dL} \\
\mathrm{Hb}<10.0 \mathrm{~g} / \mathrm{dL}+ \\
\text { symptoms }\end{array}$ & Yes & $\begin{array}{l}\text { Clamped for } 2 \\
\mathrm{~h}\end{array}$ \\
\hline Prakash et al. [26] & $\begin{array}{l}\text { LMWH } \\
\text { Calf pump }\end{array}$ & $\begin{array}{l}\text { Doppler ultrasound } \\
\text { Chest } C T\end{array}$ & Cemented & $\mathrm{Hb}<8.0 \mathrm{~g} / \mathrm{dL}$ & Yes & $\begin{array}{l}\text { Clamped for } \\
30 \mathrm{~min}\end{array}$ \\
\hline Song et al. [35] & $\mathrm{LMWH}$ in high-risk patient & $\begin{array}{l}\text { Doppler ultrasound } \\
\text { Chest CT }\end{array}$ & Cemented & $\mathrm{Hb}<8.0 \mathrm{~g} / \mathrm{dL}$ & Yes & $\begin{array}{l}\text { Clamped for } \\
10 \text { min }\end{array}$ \\
\hline Stowers et al. [24] & Aspirin & Clinical examination & Cemented & $\begin{array}{l}\mathrm{Hb}<8.0 \mathrm{~g} / \mathrm{dL} \\
\mathrm{Hb}<10.0 \mathrm{~g} / \mathrm{dL}+ \\
\text { symptoms }\end{array}$ & Yes & No drain \\
\hline Uğurlu et al. [34] & $\begin{array}{l}\text { LMWH } \\
\text { Compression stocking }\end{array}$ & Clinical examination & Unclear & $\begin{array}{l}\mathrm{Hb}<8.0 \mathrm{~g} / \mathrm{dL} \\
\mathrm{Hb}>8.0 \mathrm{~g} / \mathrm{dL}+ \\
\text { symptoms }\end{array}$ & Yes & $\begin{array}{l}\text { Clamped for } 1 \\
\text { h }\end{array}$ \\
\hline Wang et al. $[11,23]$ & $\begin{array}{l}\text { LMWH/rivaroxaban } \\
\text { Elastic bandage }\end{array}$ & Doppler ultrasound & Cemented & $\begin{array}{l}\mathrm{Hb}<6.0 \mathrm{~g} / \mathrm{dL} \\
\mathrm{Hb}>6.0+\text { symptoms }\end{array}$ & Yes & $\begin{array}{l}\text { Clamped for } 2 \\
\mathrm{~h}\end{array}$ \\
\hline Zekcer et al. [25] & $\begin{array}{l}\text { LMWH } \\
\text { Compression stocking }\end{array}$ & Unclear & Cemented & $\mathrm{Hb}<8.0 \mathrm{~g} / \mathrm{dL}$ & Yes & Unclear \\
\hline Aggarwal et al. [39] & Aspirin & Clinical examination & Cemented & $\begin{array}{l}\mathrm{Hb}>8.0 \mathrm{~g} / \mathrm{dL}+ \\
\text { symptoms }\end{array}$ & Yes & $\begin{array}{l}\text { Clamped for } 1 \\
\mathrm{~h}\end{array}$ \\
\hline Chen et al. $[29,53]$ & $\begin{array}{l}\text { LMWH } \\
\text { Calf pumps }\end{array}$ & $\begin{array}{l}\text { Clinical examination } \\
\text { Doppler ultrasound } \\
\text { Chest } C T\end{array}$ & Cemented & $\begin{array}{l}\mathrm{Hb}<8.0 \mathrm{~g} / \mathrm{dL} \\
\mathrm{Hb}<10.0 \mathrm{~g} / \mathrm{dL}+ \\
\text { symptoms }\end{array}$ & Yes & Unclear \\
\hline Drosos et al. [38] & $\begin{array}{l}\text { LMWH } \\
\text { Compression stocking }\end{array}$ & $\begin{array}{l}\text { Clinical examination } \\
\text { Doppler ultrasound }\end{array}$ & Hybrid & $\begin{array}{l}\mathrm{Hb}<10.0 \mathrm{~g} / \mathrm{dL}+ \\
\text { symptoms }\end{array}$ & Yes & No clamp \\
\hline Keyhani et al. [42] & LMWH & Doppler ultrasound & Cemented & $\mathrm{Hb}<8.0 \mathrm{~g} / \mathrm{dL}$ & Yes & $\begin{array}{l}\text { Clamped for } 2 \\
\text { h }\end{array}$ \\
\hline May et al. [37] & $\begin{array}{l}\text { LMWH } \\
\text { Sequential compression }\end{array}$ & Clinical examination & Unclear & $\begin{array}{l}\mathrm{Hb}<7.0 \mathrm{~g} / \mathrm{dL} \\
\mathrm{Hb}<10.0 \mathrm{~g} / \mathrm{dL}+ \\
\text { symptoms }\end{array}$ & Yes & No drain \\
\hline Pinsornsak et al. & Ankle pumping exercise & Clinical examination & Cemented & $\mathrm{Hb}<10.0 \mathrm{~g} / \mathrm{dL}+$ & Yes & Clamped for 3 \\
\hline
\end{tabular}


Table 4 Surgical protocols (Continued)

\begin{tabular}{|c|c|c|c|c|c|c|}
\hline Study & Thromboprophylaxis & $\begin{array}{l}\text { DVT screening } \\
\text { method }\end{array}$ & $\begin{array}{l}\text { Prosthetic } \\
\text { properties }\end{array}$ & $\begin{array}{l}\text { Blood transfusion } \\
\text { protocol }\end{array}$ & Tourniquet & Drainage \\
\hline [37] & Early ambulation & & & symptoms & & $\mathrm{h}$ \\
\hline Tzatzairis et al. [40] & $\begin{array}{l}\text { LMWH } \\
\text { Compression stocking }\end{array}$ & $\begin{array}{l}\text { Doppler ultrasound } \\
\text { Clinical examination } \\
\text { Chest CT }\end{array}$ & Cemented & $\begin{array}{l}\mathrm{Hb}<10.0 \mathrm{~g} / \mathrm{dL}+ \\
\text { symptoms }\end{array}$ & No & $\begin{array}{l}\text { Clamped for } 1 \\
\text { h }\end{array}$ \\
\hline Aguilera et al. [43] & LMWH & Clinical examination & Cemented & $\begin{array}{l}\mathrm{Hb}<8.0 \mathrm{~g} / \mathrm{dL} \\
\mathrm{Hb}<9.0 \mathrm{~g} / \mathrm{dL}+ \\
\text { symptoms }\end{array}$ & Yes & $\begin{array}{l}\text { Clamped for } 1 \\
\mathrm{~h}\end{array}$ \\
\hline Digas et al. [44] & Tinzaparin & Clinical examination & Cemented & $\begin{array}{l}\mathrm{Hb}<8.5 \mathrm{~g} / \mathrm{dL} \\
\mathrm{Hb}<9.5 \mathrm{~g} / \mathrm{dL}+ \\
\text { symptoms }\end{array}$ & Yes & $\begin{array}{l}\text { Clamped for } 3 \\
\text { h }\end{array}$ \\
\hline Öztaş et al. [45] & LMWH & Clinical examination & Unclear & $\begin{array}{l}\mathrm{Hb}<8.0 \mathrm{~g} / \mathrm{dL} \\
\mathrm{Hb}<10.0 \mathrm{~g} / \mathrm{dL}+ \\
\text { symptoms }\end{array}$ & Yes & $\begin{array}{l}\text { Clamped for } \\
30 \text { min }\end{array}$ \\
\hline $\begin{array}{l}\text { Gomez-Barrena } \\
\text { et al. [46] }\end{array}$ & LMWH & $\begin{array}{l}\text { Clinical examination } \\
\text { Doppler ultrasound }\end{array}$ & Cemented & $\begin{array}{l}\mathrm{Hb}<8.0 \mathrm{~g} / \mathrm{dL} \\
\mathrm{Hb}<10.0 \mathrm{~g} / \mathrm{dL}+ \\
\text { symptoms }\end{array}$ & Yes & $\begin{array}{l}\text { Clamped for } 2 \\
\mathrm{~h}\end{array}$ \\
\hline Patel et al. [47] & LMWH & $\begin{array}{l}\text { Doppler ultrasound } \\
\text { Chest } C T\end{array}$ & Unclear & $\begin{array}{l}\mathrm{Hb}<8.0 \mathrm{~g} / \mathrm{dL}+ \\
\text { symptoms }\end{array}$ & Yes & Yes \\
\hline Sarzaeem et al. [48] & Unclear & Unclear & Cemented & $\begin{array}{l}\mathrm{Hb}<8.0 \mathrm{~g} / \mathrm{dL} \\
\mathrm{Hb}<10.0 \mathrm{~g} / \mathrm{dL}+ \\
\text { symptoms }\end{array}$ & Yes & $\begin{array}{l}\text { Clamped for } 1 \\
\mathrm{~h}\end{array}$ \\
\hline Soni et al. [49] & $\begin{array}{l}\text { LMWH } \\
\text { Ankle pumping exercise }\end{array}$ & Clinical examination & Cemented & $\mathrm{Hb}<8.0 \mathrm{~g} / \mathrm{dL}$ & Yes & $\begin{array}{l}\text { Clamped for } 1 \\
\mathrm{~h}\end{array}$ \\
\hline Seo et al. [50] & Unclear & Unclear & Cemented & $\begin{array}{l}\mathrm{Hb}<8.0 \mathrm{~g} / \mathrm{dL} \\
\mathrm{Hb}<10.0 \mathrm{~g} / \mathrm{dL}+ \\
\text { symptoms }\end{array}$ & Yes & No drain \\
\hline Maniar et al. [51] & $\begin{array}{l}\text { LMWH } \\
\text { Ankle pumping exercise } \\
\text { Compression stocking }\end{array}$ & $\begin{array}{l}\text { Clinical examination } \\
\text { Doppler ultrasound }\end{array}$ & Cemented & $\begin{array}{l}\mathrm{Hb}<8.5 \mathrm{~g} / \mathrm{dL} \\
\mathrm{Hb}<10.0 \mathrm{~g} / \mathrm{dL}+ \\
\text { symptoms }\end{array}$ & Yes & $\begin{array}{l}\text { Clamped for } 2 \\
\mathrm{~h}\end{array}$ \\
\hline
\end{tabular}

$\mathrm{Hb}$ hemoglobin, $L M W H$ low-molecular-weight heparin

\section{Blood transfusion rate}

Twenty-eight studies involving 3270 patients had data on blood transfusion. Transfusions were reported as $109 / 1664$ (6.6\%) in the IV group and 99/1606 (6.2\%) in the IA group. Only 25 studies with 2950 patients were included in our meta-analysis, while the other three studies reported no transfusion event. The risk of a blood transfusion was similar between the two groups $(\mathrm{OR}=0.93,95 \% \mathrm{CI}=0.69$ to $1.24, P=0.62)$, and the data showed low heterogeneity $\left(P=0.54, I^{2}=0 \%\right)$.

\section{Complications}

In our study, certain complications were our concern, including DVT, PE, wound complications, and other adverse events. In all, 33 studies involving 3807 patients mentioned data of complications. The incidence of complications was mentioned as 77/1946 (4.0\%) in the IV group and $77 / 1861(4.1 \%)$ in the IA group. In these 33 studies, 13 of them reported no complication, so only 20 studies with 2594 patients were included in the metaanalysis. The risk was the same between the two groups
$(\mathrm{OR}=1.00,95 \% \mathrm{CI}=0.72$ to $1.39, P=0.98)$ with low heterogeneity $\left(P=0.47, I^{2}=0 \%\right)$.

In subgroup analysis, complications were classified into four types: DVT, PE, wound complications, and other adverse events. All subgroups showed insignificant differences between the IV and IA groups.

There were 23 DVT events reported in ten studies among all 33 studies. Pooled results showed a similar risk $(\mathrm{OR}=0.92,95 \% \mathrm{CI}=0.44$ to $1.92, P=0.83)$ with low heterogeneity $\left(P=0.84, I^{2}=0 \%\right)$. Both the IV and IA groups had four PE events reported in three studies $[15,24,37]$. The risk of PE was similar between the IV group and IA group (OR $=1.02$, 95\% $\mathrm{CI}=0.25$ to $4.20, P=0.98)$ with low heterogeneity $\left(P=0.81, I^{2}=0 \%\right)$.

Wound complications included infection, necrosis, delay healing, and dehiscence. There were 58 wound complications reported in 14 studies. A fixed-effects model was used due to low heterogeneity $(P=0.39$, $I^{2}=6 \%$ ), and a similar risk of wound complications was shown in two groups $(\mathrm{OR}=0.95,95 \% \mathrm{CI}=0.58$ to $1.55, P=0.83$ ). 
Table 5 Methodological quality of included studies

\begin{tabular}{|c|c|c|c|c|c|c|c|}
\hline Study & $\begin{array}{l}\text { Quality } \\
\text { score }\end{array}$ & Random generation sequence & $\begin{array}{l}\text { Allocation } \\
\text { concealment }\end{array}$ & Blind & $\begin{array}{l}\text { Incomplete } \\
\text { outcome data }\end{array}$ & $\begin{array}{l}\text { Selective } \\
\text { reporting }\end{array}$ & $\begin{array}{l}\text { Other } \\
\text { biases }\end{array}$ \\
\hline $\begin{array}{l}\text { Jules-Elysee et al. } \\
{[14]}\end{array}$ & 7 & $\begin{array}{l}\text { Computer-generated randomization } \\
\text { schedule }\end{array}$ & Unclear & Yes & No & No & No \\
\hline $\begin{array}{l}\text { Laoruengthana } \\
\text { et al. [13] }\end{array}$ & 8 & Computer-generated numbers & Concealed envelope & Yes & No & No & No \\
\hline $\begin{array}{l}\text { Zhang et al. [15, } \\
\text { 52] }\end{array}$ & 8 & Randomized numbers table & $\begin{array}{l}\text { Labeled with } \\
\text { numbering code }\end{array}$ & Yes & No & No & No \\
\hline Abdel et al. [20] & 6 & Randomized but unknown method & Unclear & Yes & No & No & No \\
\hline Ahmed et al. [17] & 6 & The lottery method & Unclear & No & No & No & No \\
\hline $\begin{array}{l}\text { López-Hualda et al } \\
\text { [21] }\end{array}$ & 5 & Randomized but unknown method & Unclear & No & No & No & No \\
\hline George et al. [16] & 8 & Computer-generated numbers & Concealed envelope & Yes & No & No & No \\
\hline $\begin{array}{l}\text { Subramanyam } \\
\text { et al. [19] }\end{array}$ & 8 & Computer-generated numbers & Concealed envelope & Yes & No & No & No \\
\hline Wei et al. [18] & 8 & Randomized numbers table & Concealed envelope & Yes & No & No & No \\
\hline Goyal et al. [36] & 8 & Computer-generated numbers & Concealed envelope & Yes & No & No & No \\
\hline Lacko et al. [22] & 6 & Computer-generated numbers & Unclear & No & No & No & No \\
\hline Maniar et al. [33] & 8 & $\begin{array}{l}\text { Randomly drawing sealed envelope } \\
\text { from container }\end{array}$ & Concealed envelope & Yes & No & No & No \\
\hline Prakash et al. [26] & 7 & Randomized but unknown method & Concealed envelope & Yes & No & No & No \\
\hline Song et al. [35] & 8 & Computer-generated numbers & Concealed envelope & Yes & No & No & No \\
\hline Stowers et al. [24] & 8 & Block randomization & Concealed envelope & Yes & No & No & No \\
\hline Uğurlu et al. [34] & 5 & Randomized but unknown method & Unclear & No & No & No & No \\
\hline $\begin{array}{l}\text { Wang et al. [11, } \\
\text { 23] }\end{array}$ & 8 & $\begin{array}{l}\text { Randomly drawing sealed envelope } \\
\text { from container }\end{array}$ & Concealed envelope & Yes & No & No & No \\
\hline Zekcer et al. [25] & 8 & $\begin{array}{l}\text { Randomly drawing sealed envelope } \\
\text { from container }\end{array}$ & Concealed envelope & Yes & No & No & No \\
\hline $\begin{array}{l}\text { Aggarwal et al. } \\
\text { [39] }\end{array}$ & 8 & Computer-generated numbers & Concealed envelope & Yes & No & No & No \\
\hline Chen et al. $[29,53]$ & 8 & Randomized numbers table & Concealed envelope & Yes & No & No & No \\
\hline Drosos et al. [38] & 8 & $\begin{array}{l}\text { Stratified randomization by } \\
\text { minimization }\end{array}$ & Concealed envelope & Yes & No & No & No \\
\hline Keyhani et al. [42] & 5 & Randomized but unknown method & Unclear & No & No & No & No \\
\hline May et al. [37] & 7 & Randomized numbers table & Unclear & Yes & No & No & No \\
\hline $\begin{array}{l}\text { Pinsornsak et al. } \\
\text { [37] }\end{array}$ & 7 & Randomized but unknown method & Concealed envelope & Yes & No & No & No \\
\hline Tzatzairis et al. [40] & 6 & $\begin{array}{l}\text { Stratified randomization by } \\
\text { minimization }\end{array}$ & Unclear & No & No & No & No \\
\hline Aguilera et al. [43] & 7 & Randomized numbers table & Unclear & Yes & No & No & No \\
\hline Digas et al. [44] & 7 & Randomized but unknown method & Concealed envelope & Yes & No & No & No \\
\hline Öztaş et al. [45] & 5 & Randomized but unknown method & Unclear & No & No & No & No \\
\hline $\begin{array}{l}\text { Gomez-Barrena } \\
\text { et al. [46] }\end{array}$ & 7 & Randomized but unknown method & Concealed envelope & Yes & No & No & No \\
\hline Patel et al. [47] & 7 & Excel's randomization & Unclear & Yes & No & No & No \\
\hline $\begin{array}{l}\text { Sarzaeem et al. } \\
\text { [48] }\end{array}$ & 7 & Randomized numbers table & Unclear & Yes & No & No & No \\
\hline Soni et al. [49] & 6 & Computer-generated numbers & Unclear & No & No & No & No \\
\hline Seo et al. [50] & 7 & Randomized numbers table & Unclear & Yes & No & No & No \\
\hline Maniar et al. [51] & 8 & $\begin{array}{l}\text { Randomly drawing sealed envelope } \\
\text { from container }\end{array}$ & Concealed envelope & Yes & No & No & No \\
\hline
\end{tabular}


Table 6 Results of meta-analysis and subgroup analyses

\begin{tabular}{|c|c|c|c|c|c|c|}
\hline Variables & Studies $(n)$ & Patients $(n)$ & $P$ value & Incidence: OR/MDs (95\% Cl) & Heterogeneity: $P$ value $\left(I^{2}\right)$ & Model \\
\hline \multirow[t]{2}{*}{ Total blood loss (TBL) } & 18 & 1656 & $<0.001^{*}$ & 63.99 (27.81 to 100.16$)$ & $<0.001^{*}(81 \%)$ & Random \\
\hline & 13 & 1197 & $<0.001^{*}$ & 33.38 (19.24 to 47.51) & $0.34(11 \%)$ & Fixed \\
\hline Drain output & 17 & 1494 & $0.03^{*}$ & 28.44 (2.61 to 54.27$)$ & $<0.001 *(93 \%)$ & Random \\
\hline Clamp $<2 \mathrm{~h}$ & 7 & 607 & $0.03^{*}$ & 51.47 (6.02 to 96.92$)$ & $<0.001^{*}(92 \%)$ & Random \\
\hline Clamp $\geq 2 \mathrm{~h}$ & 10 & 887 & 0.51 & $12.40(-24.85$ to 49.65$)$ & $<0.001 *(89 \%)$ & Random \\
\hline Hidden blood loss (HBL) & 6 & 640 & 0.83 & $7.57(-60.34$ to 75.47$)$ & $0.006(69 \%)$ & Random \\
\hline Hemoglobin $(\mathrm{Hb})$ fall & 19 & 1749 & 0.79 & $-0.02(-0.20$ to 0.16$)$ & $<0.001 *(87 \%)$ & Random \\
\hline \multirow[t]{2}{*}{ POD1 } & 10 & 1052 & 0.07 & $-0.34(-0.70$ to 0.02$)$ & $<0.001^{*}(91 \%)$ & Random \\
\hline & 8 & 839 & 0.86 & $-0.01(-0.11$ to 0.13$)$ & $0.14(36 \%)$ & Fixed \\
\hline \multirow[t]{2}{*}{ POD2 } & 8 & 701 & 0.37 & $0.17(-0.20$ to 0.53$)$ & $<0.001 *(82 \%)$ & Random \\
\hline & 6 & 531 & 0.36 & $-0.08(-0.25$ to 0.09$)$ & $0.11(44 \%)$ & Fixed \\
\hline POD3+ & 6 & 637 & $0.001^{*}$ & 0.24 (0.09 to 0.39 ) & $0.18(34 \%)$ & Fixed \\
\hline Transfusion rate & 25 & 2950 & 0.62 & 0.93 (0.69 to 1.24$)$ & $0.54(0 \%)$ & Fixed \\
\hline Complications & 20 & 2594 & 0.98 & 1.00 (0.72 to 1.39$)$ & $0.47(0 \%)$ & Fixed \\
\hline DVT & 10 & 1641 & 0.83 & 0.92 (0.44 to 1.92 ) & $0.84(0 \%)$ & Fixed \\
\hline PE & 3 & 342 & 0.98 & 1.02 (0.25 to 4.20$)$ & $0.81(0 \%)$ & Fixed \\
\hline Wound complications & 14 & 1465 & 0.83 & 0.95 (0.58 to 1.55$)$ & $0.39(6 \%)$ & Fixed \\
\hline Other adverse events & 13 & 1899 & 0.69 & 1.10 (0.68 to 1.80$)$ & $0.42(2 \%)$ & Fixed \\
\hline Length of stay & 7 & 748 & 0.33 & $0.07(-0.07$ to 0.22$)$ & $0.35(11 \%)$ & Fixed \\
\hline Tourniquet time & 9 & 816 & 0.19 & $-1.22(-3.06$ to 0.62$)$ & $0.74(0 \%)$ & Fixed \\
\hline
\end{tabular}

$P O D$ postoperative day, DVT deep vein thrombosis $* 0.05$

Other adverse events were reported in 65 patients of 13 studies. Zhang et al. [15] reported 14 patients with idiopathic venous thromboembolism, and Wang et al. [23] reported one patient with intramuscular vein thrombosis. Besides, Abdel et al. [20] reported one patient with a thrombotic cerebrovascular accident. Functional disorders, such as stiffness, vomiting, nausea, dizziness, constipation, and paresthesia, were also reported in several studies $[36,43,46]$. A similar risk was shown $(\mathrm{OR}=1.10$, $95 \% \mathrm{CI}=0.68$ to $1.80, P=0.69)$ with low heterogeneity $(P$ $\left.=0.42, I^{2}=2 \%\right)$ between IA and IV.

\section{Length of hospital stay}

Seven studies involving 748 patients reported data on length of hospital stay. Because of low heterogeneity $(P$ $=0.35, I^{2}=11 \%$ ), we used a fixed-effects model for

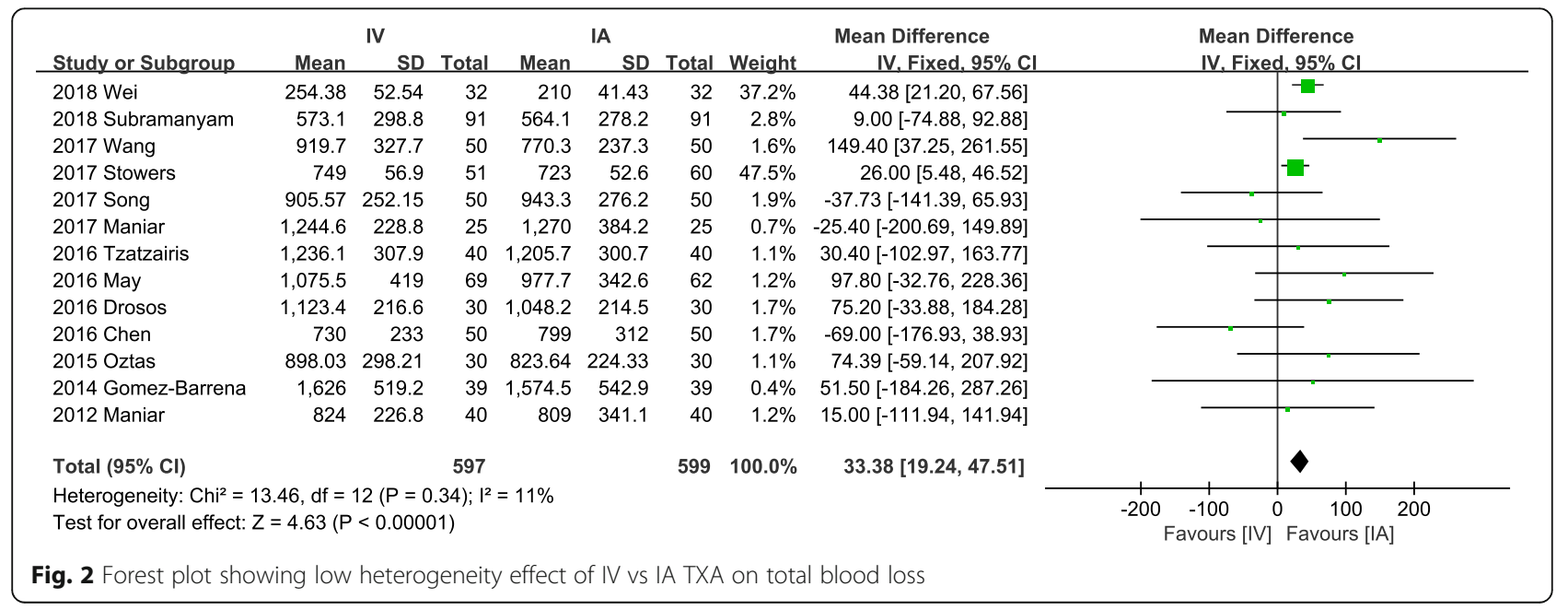




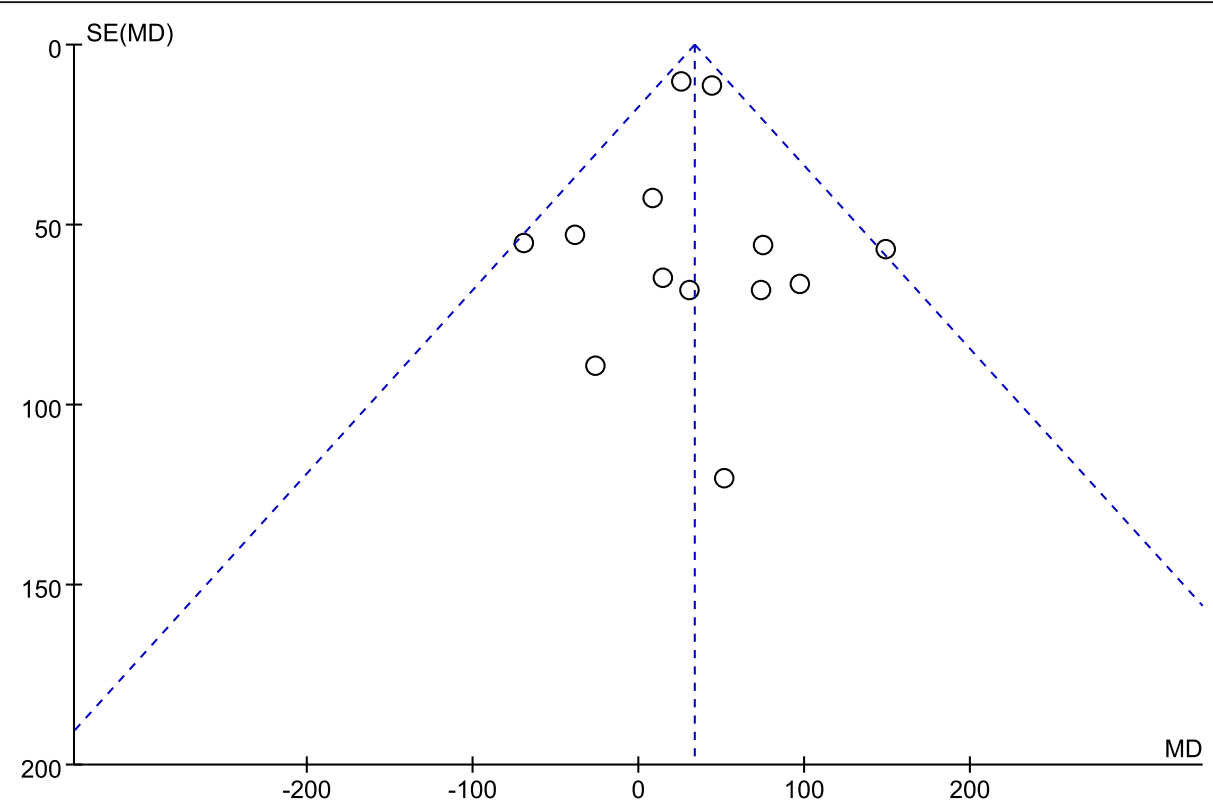

Fig. 3 Funnel plot of TBL shows low publication bias

analysis. There was no significant difference in this comparison $(\mathrm{MD}=0.07,95 \% \mathrm{CI}=-0.07$ to $0.22, P=0.33$ ).

\section{Duration of tourniquet application}

Nine studies including 815 patients reported data of tourniquet time. A fixed-effects model was used for analysis due to the low heterogeneity $\left(P=0.74, I^{2}=0 \%\right)$. It did not show a statistical difference between the two groups $(\mathrm{MD}=-1.22,95 \% \mathrm{CI}=-3.06$ to $0.62, P=$ $0.19)$.

\section{Discussion}

The most important finding in our study is that the difference of TBL and drain output between IV and IA administration is supported by newly added RCTs. Based on available evidences, the IA group shows significant superiority over the IV group regarding TBL, drain output, and POD3+ Hb fall. Besides, this study suggests that there exists no statistical difference on HBL, POD1 and POD2 $\mathrm{Hb}$ fall, incidence of blood transfusion, length of hospital stay, and time of tourniquet application between the two groups.

As an antifibrinolytic agent, TXA is a synthetic derivative of the amino acid lysine which competitively blocks the lysine-binding sites in the plasmin and plasminogen activator molecules, thereby preventing dissolution of the fibrin clot [54]. A previous study [6], which included 23,236 patients undergoing primary TKA, proved that TXA application was associated with decreased blood loss and transfusion risk without noticeably increased risk of complications. Besides, it could also reduce the risk of venous thromboembolism [6]. Several previous studies have compared IV and IA administration in TKA: Xie et al. [55] included 18 RCTs and found no significant difference between IV and IA. Gianakos et al. [12] included 18 RCTs and 5 non-RCTs, and they found significant differences regarding TBL and drain output between IV and IA. However, it was a study of high heterogeneity. Therefore, we performed this meta-analysis with more newly published RCTs. Moreover, subgroup analysis and sensitivity analysis were performed to reach a more convincing conclusion.

In our study, IA administration shows significant superiority on the TBL to IV group $(\mathrm{MD}=33.38, P<$ 0.001). A previous study indicated easier administration of topical TXA with a maximum concentration at the bleeding site and minimal systemic absorption [53], and therefore, topical application may deliver better blood loss control theoretically. The IA group also shows significant superiority on drain output $(\mathrm{MD}=28.44, P=$ $0.03)$. The difference is more significant when the drainage tube is clamped postoperatively less than $2 \mathrm{~h}(\mathrm{MD}=$ 51.47, $P=0.03$ ). However, when the drainage tube is clamped over $2 \mathrm{~h}$ after surgery, there exists no statistical difference between them $(P=0.51)$. It is possibly due to a higher concentration of TXA and longer contact time in the IA approach.

There exists a significant difference on POD3+ $\mathrm{Hb}$ fall $(\mathrm{MD}=0.24, P=0.001)$, while POD1 $(P=0.86)$ and POD2 $\mathrm{Hb}$ fall $(P=0.36)$ show no noticeable difference between the two groups. POD3+ $\mathrm{Hb}$ fall is usually caused by HBL [55]. However, due to the limited data, 


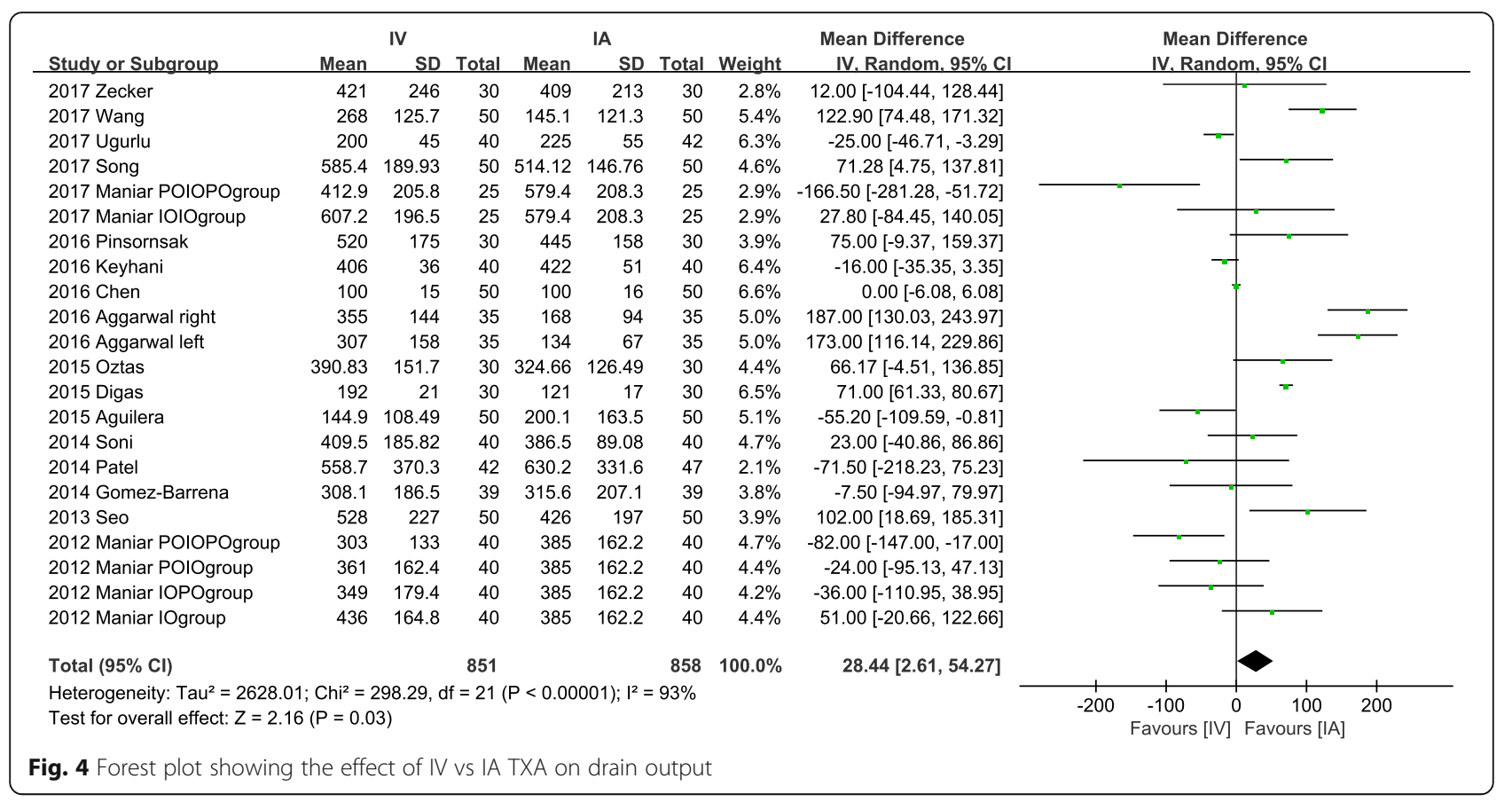

there exists no difference on HBL $(P=0.83)$. Besides, IV administration of TXA has a maximal systemic absorption which may result in a shorter efficacy time in theory [56]. Therefore, it is a reasonable explanation of similar effects on POD1 and POD2, and a better result in the IA group on POD3+.

Fillingham et al. [5] published a clinical guideline of TXA application in joint replacement, but no optimal approach was recommended. In contrast, in our study, IA was found to be of superior value in light of the recently published RCT results. Although we have not compared IA with oral or combined administration, future clinical trials might validate our findings and possibly influence the revision of the clinical guideline of TXA. Besides, Fillingham et al. [5] also admitted dosage amount and multiple doses of TXA did not significantly affect the blood loss. However, several recent studies had different conclusions. Tzatzairis et al. [57] made a comparison between one to three doses of $15 \mathrm{mg} / \mathrm{kg}$ TXA intravenously and concluded that the three-dose group displayed better outcome. Lei et al. [58] reach the same conclusion by comparing $20 \mathrm{mg} / \mathrm{kg}$ and 60 $\mathrm{mg} / \mathrm{kg}$ TXA intravenously. Moreover, Zhang et al. [52] even reported a better outcome of six-dose IV TXA. Besides, Tammachote et al. [59] compared high dosage $(3 \mathrm{~g})$ with low dosage $(0.5 \mathrm{~g})$ for IA TXA and also found a better outcome of high dosage. All the results of recent RCTs favor high-dose administration of TXA. Although TXA dosage and timing were popular topics, there is no meta-analysis about them by now. In our meta-analysis, there existed no standard dosage protocol for included studies (Table 3): In the IV group, $52.9 \%$ of the studies (18 studies) used a weight-based dosage (10 to $20 \mathrm{mg} / \mathrm{kg}$ ) and the rest $47.1 \%$ of the studies (16 studies) chose a standard dosage $(0.5$ to $1.5 \mathrm{~g})$. In the IA group, only $5.9 \%$ of the studies (2 studies) used a weight-based dosage $(15 \mathrm{mg} / \mathrm{kg})$ and the rest $94.1 \%$ of the studies (32 studies) chose a standard dosage (0.75 to $3 \mathrm{~g}$ ). However, restricted by limited data, we did not perform a subgroup analysis for TXA dose and timing.

Advantages of our study include substantial highquality RCTs (Table 5) and adequate analysis. $73.5 \%$ of the studies (25 studies) have detailed random generation sequence, and $55.9 \%$ of the studies (19 studies) have adequate allocation concealment. Besides, $73.5 \%$ of the studies (25 studies) are recent studies (published after 2015). Our analyzing methods are subgroup analysis and sensitivity analysis when the previous analysis has high heterogeneity.

There are several limitations in our studies. Firstly, the inherent bias in different studies because of the inconsistent threshold for blood transfusion cannot be overlooked. Besides, the DVT rate might be influenced by the inclusion criteria, and the RCT of TXA in a DVT high-risk population might be required to validate our findings. Furthermore, repeated dose seemed a better choice than a single dose in both IV and IA administration [52, 57-59], and therefore, different methods of administration may influence the result. Lastly, data for 
HBL, length of hospital stay, and duration of tourniquet application are limited for analysis, and costeffectiveness remains to be investigated.

\section{Conclusion}

IA administration of TXA is superior to IV TXA in patients receiving primary TKA regarding the performance on TBL, drain output, and POD3+ Hb fall, without noticeably increased risk of complications. Therefore, IA administration should be the preferred approach in clinical practice.

\section{Abbreviations \\ IA: Intra-articular; IV: Intravenous; TXA: Tranexamic acid; TKA: Total knee arthroplasty; Hb: Hemoglobin; OA: Osteoarthritis; TBL: Total blood loss; HBL: Hidden blood loss; POD: Postoperative day; DVT: Deep vein thrombosis; PE: Pulmonary embolism; LMWH: Low-molecular-weight heparin; RCT: Randomized controlled trial; BMl: Body mass index; OR: Odds ratio; MD: Mean difference; $\mathrm{Cl}$ : Confidence interval}

\section{Acknowledgements}

Not applicable.

\section{Authors' contributions}

$\mathrm{PH}^{*}$ is in charge of the main idea and is the guarantor of the integrity of the entire study; $J$ and RKL contributed equally to this manuscript. $J$ and RKL are in charge of the study concepts, design, manuscript preparation, and editing; $\mathrm{PH}$ and $\mathrm{SR}$ are in charge of the language polishing and the grammar revision; $\mathrm{RHZ}$ and $\mathrm{XT}$ are in charge of the collection of the data. All authors read and approved the final manuscript.

\section{Funding}

This study is supported by the Nature Science Foundation of Hubei Province [2018CFB590]. The foundation had no roles in the study design, data collection and analysis, decision to publish, or preparation of the manuscript.

\section{Availability of data and materials}

We state that the data will not be shared because all the raw data are present in the figures included in the article.

\section{Ethics approval and consent to participate}

Not applicable.

\section{Consent for publication}

Not applicable.

\section{Competing interests}

The authors declare that they have no competing interests.

\section{Author details}

'Department of Orthopaedic Surgery, Union Hospital, Tongji Medical College, Huazhong University of Science and Technology, Wuhan 430022, China.

${ }^{2}$ First School of Clinical Medicine, Tongji Medical College, Huazhong University of Science and Technology, Wuhan, China. ${ }^{3}$ Department of Orthopaedics and Trauma Surgery, National Trauma Center, National Academy of Medical Sciences, Mahankal, Kathmandu, Nepal.

Received: 20 June 2020 Accepted: 24 November 2020 Published online: 02 December 2020

\section{References}

1. Kurtz SM, Ong KL, Lau E, et al. International survey of primary and revision total knee replacement. Int Orthop. 2011:35(12):1783-9.

2. Kurtz S, Ong K, Lau E, Mowat F, Halpern M. Projections of primary and revision hip and knee arthroplasty in the United States from 2005 to 2030. J Bone Joint Surg Am. 2007:89(4):780-5.

3. Hussain SM, Neilly DW, Baliga S, Patil S, Meek R. Knee osteoarthritis: a review of management options. Scott Med J. 2016;61(1):7-16.
4. Spahn DR. Anemia and patient blood management in hip and knee surgery: a systematic review of the literature. Anesthesiology. 2010;113:482-95.

5. Fillingham YA, Ramkumar DB, Jevsevar DS, et al. Tranexamic acid use in total joint arthroplasty: the clinical practice guidelines endorsed by the American Association of Hip and Knee Surgeons, American Society of Regional Anesthesia and Pain Medicine, American Academy of Orthopaedic Surgeons, Hip Society, and Knee Society. J Arthroplasty. 2018;33(10):3065-9.

6. Hallstrom B, Singal B, Cowen ME, Roberts KC, Hughes RE. The Michigan experience with safety and effectiveness of tranexamic acid use in hip and knee arthroplasty. J Bone Joint Surg Am. 2016;98(19):1646-55.

7. Fillingham YA, Ramkumar DB, Jevsevar DS, et al. The safety of tranexamic acid in total joint arthroplasty: a direct meta-analysis. J Arthroplasty. 2018; 33(10):3070-82

8. Fillingham YA, Ramkumar DB, Jevsevar DS, et al. The efficacy of tranexamic acid in total knee arthroplasty: a network meta-analysis. J Arthroplasty. 2018; 33(10):3090-8

9. Guo P, He Z, Wang Y, et al. Efficacy and safety of oral tranexamic acid in total knee arthroplasty: a systematic review and meta-analysis. Medicine (Baltimore). 2018;97(18):587.

10. Liu W, Yang C, Huang X, Liu R. Tranexamic acid reduces occult blood loss, blood transfusion, and improves recovery of knee function after total knee arthroplasty: a comparative study. J Knee Surg. 2018;31(3):239-46.

11. Wang S, Gao X, An Y. Topical versus intravenous tranexamic acid in total knee arthroplasty: a meta-analysis of randomized controlled trials. Int Orthop. 2017;41(4):739-48.

12. Gianakos AL, Hurley ET, Haring RS, Yoon RS, Liporace FA. Reduction of blood loss by tranexamic acid following total hip and knee arthroplasty: a meta-analysis. JBJS Rev. 2018;6(5):1.

13. Laoruengthana A, Rattanaprichavej P, Rasamimongkol S, Galassi M, Weerakul S, Pongpirul K. Intra-articular tranexamic acid mitigates blood loss and morphine use after total knee arthroplasty. A randomized controlled trial. J Arthroplasty. 2019;34(5):877-81.

14. Jules-Elysee KM, Tseng A, Sculco TP, et al. Comparison of topical and intravenous tranexamic acid for total knee replacement: a randomized double-blinded controlled study of effects on tranexamic acid levels and thrombogenic and inflammatory marker levels. J Bone Joint Surg Am. 2019; 101(23):2120-8.

15. Zhang YM, Yang B, Sun XD, Zhang Z. Combined intravenous and intraarticular tranexamic acid administration in total knee arthroplasty for preventing blood loss and hyperfibrinolysis: a randomized controlled trial. Medicine (Baltimore). 2019;98(7):e14458.

16. George J, Eachempati KK, Subramanyam KN, Gurava Reddy AV. The comparative efficacy and safety of topical and intravenous tranexamic acid for reducing perioperative blood loss in total knee arthroplasty - a randomized controlled non-inferiority trial. Knee. 2018;25(1):185-91.

17. Ahmed S, Ahmed A, Ahmad S, Atiq-uz-Zaman, Javed S, Aziz A. Blood loss after intraarticular and intravenous tranexamic acid in total knee arthroplasty. J Pak Med Assoc. 2018;68(10):1434-7.

18. Wei W, Dang S, Duan D, Wei L. Comparison of intravenous and topical tranexamic acid in total knee arthroplasty. BMC Musculoskelet Disord. 2018;19(1):191-5.

19. Subramanyam KN, Khanchandani P, Tulajaprasad PV, Jaipuria J, Mundargi AV. Efficacy and safety of intra-articular versus intravenous tranexamic acid in reducing perioperative blood loss in total knee arthroplasty: a prospective randomized double-blind equivalence trial. Bone Joint J. 2018; $100 \mathrm{~B}(2): 152-60$

20. Abdel MP, Chalmers BP, Taunton MJ, et al. Intravenous versus topical tranexamic acid in total knee arthroplasty: both effective in a randomized clinical trial of 640 patients. J Bone Joint Surg Am. 2018;100(12):1023-9.

21. López-Hualda Á, Dauder-Gallego C, Ferreño-Márquez D, Martínez-Martín J. Efficacy and safety of topical tranexamic acid in knee arthroplasty. Med Clin (Barc). 2018;151(11):431-4.

22. Lacko M, Cellar R, Schreierova D, Vasko G. Comparison of intravenous and intra-articular tranexamic acid in reducing blood loss in primary total knee replacement. Eklem Hastalik Cerrahisi. 2017;28(2):64-71.

23. Wang J, Wang Q, Zhang X, Wang Q. Intra-articular application is more effective than intravenous application of tranexamic acid in total knee arthroplasty: a prospective randomized controlled trial. J Arthroplasty. 2017; 32(11):3385-9.

24. Stowers MDJ, Aoina J, Vane A, Poutawera V, Hill AG, Munro JT. Tranexamic acid in knee surgery study-a multicentered, randomized, controlled trial. J Arthroplasty. 2017;32(11):3379-84. 
25. Zekcer A, Priori RD, Tieppo C, Silva RSD, Severino NR. Comparative study of topical vs. intravenous tranexamic acid regarding blood loss in total knee arthroplasty. Rev Bras Ortop. 2017:52(5):589-95.

26. Prakash J, Seon JK, Park YJ, Jin C, Song EK. A randomized control trial to evaluate the effectiveness of intravenous, intra-articular and topical wash regimes of tranexamic acid in primary total knee arthroplasty. J Orthop Surg (Hong Kong). 2017;25(1):1-7.

27. Moher D, Shamseer L, Clarke M, et al. Preferred reporting items for systematic review and meta-analysis protocols (PRISMA-P) 2015 statement. Syst Rev. 2015;4(1):1.

28. Gross JB. Estimating allowable blood loss: corrected for dilution. Anesthesiology. 1983;58:277-80.

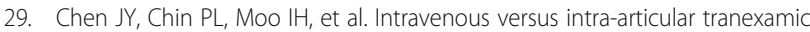
acid in total knee arthroplasty: a double-blinded randomised controlled noninferiority trial. Knee. 2016;23(1):152-6.

30. Higgins JPT, Thomas J, Chandler J, et al. Cochrane handbook for systematic reviews of interventions version 6.0 (updated 2019). Cochrane, 2019. Available from www.training.cochrane.org/handbook. Accessed $16 \mathrm{Mar}$ 2020.

31. Oremus M, Wolfson C, Perrault A, Demers L, Momoli F, Moride Y. Interrater reliability of the modified Jadad quality scale for systematic reviews of Alzheimer's disease drug trials. Dement Geriatr Cogn Disord. 2001;12(3):232-6.

32. Higgins JP, Thompson SG, Deeks JJ, Altman DG. Measuring inconsistency in meta-analyses. BMJ. 2003;327(7414):557-60.

33. Maniar RN, Singhi T, Patil A, Kumar G, Maniar P, Singh J. Optimizing effectivity of tranexamic acid in bilateral knee arthroplasty - a prospective randomized controlled study. Knee. 2017;24(1):100-6.

34. Uğurlu M, Aksekili MA, Çağlar C, Yüksel K, Şahin E, Akyol M. Effect of topical and intravenously applied tranexamic acid compared to control group on bleeding in primary unilateral total knee arthroplasty. J Knee Surg. 2017; 30(2):152-7.

35. Song EK, Seon JK, Prakash J, Seol YJ, Park YJ, Jin C. Combined administration of IV and topical tranexamic acid is not superior to either individually in primary navigated TKA. J Arthroplasty. 2017;32(1):37-42.

36. Goyal N, Chen DB, Harris IA, Rowden NJ, Kirsh G, MacDessi SJ. Intravenous vs intra-articular tranexamic acid in total knee arthroplasty: a randomized, double-blind trial. J Arthroplasty. 2017;32(1):28-32.

37. May JH, Rieser GR, Williams CG, Markert RJ, Bauman RD, Lawless MW. The assessment of blood loss during total knee arthroplasty when comparing intravenous vs intracapsular administration of tranexamic acid. $J$ Arthroplasty. 2016;31(11):2452-7.

38. Drosos $\mathrm{Gl}$, Ververidis A, Valkanis $\mathrm{C}$, et al. A randomized comparative study of topical versus intravenous tranexamic acid administration in enhanced recovery after surgery (ERAS) total knee replacement. J Orthop. 2016;13(3):127-31.

39. Aggarwal AK, Singh N, Sudesh P. Topical vs intravenous tranexamic acid in reducing blood loss after bilateral total knee arthroplasty: a prospective study. J Arthroplasty. 2016;31(7):1442-8.

40. Tzatzairis TK, Drosos GI, Kotsios SE, Ververidis AN, Vogiatzaki TD, Kazakos KI Intravenous vs topical tranexamic acid in total knee arthroplasty without tourniquet application: a randomized controlled study. J Arthroplasty. 2016; 31(11):2465-70

41. Pinsornsak P, Rojanavijitkul S, Chumchuen S. Peri-articular tranexamic acid injection in total knee arthroplasty: a randomized controlled trial. BMC Musculoskelet Disord. 2016;17:313.

42. Keyhani S, Esmailiejah AA, Abbasian MR, Safdari F. Which route of tranexamic acid administration is more effective to reduce blood loss following total knee arthroplasty? Arch Bone Jt Surg. 2016;4(1):65-9.

43. Aguilera X, Martínez-Zapata MJ, Hinarejos P, et al. Topical and intravenous tranexamic acid reduce blood loss compared to routine hemostasis in total knee arthroplasty: a multicenter, randomized, controlled trial. Arch Orthop Trauma Surg. 2015;135(7):1017-25.

44. Digas G, Koutsogiannis I, Meletiadis G, Antonopoulou E, Karamoulas V, Bikos C. Intra-articular injection of tranexamic acid reduce blood loss in cemented total knee arthroplasty. Eur J Orthop Surg Traumatol. 2015;25(7):1181-8.

45. Öztaş S, Öztürk A, Akalin Y, et al. The effect of local and systemic application of tranexamic acid on the amount of blood loss and allogeneic blood transfusion after total knee replacement. Acta Orthop Belg. 2015;81(4):698-707.

46. Gomez-Barrena E, Ortega-Andreu M, Padilla-Eguiluz NG, Pérez-Chrzanowska $H$, Figueredo-Zalve R. Topical intra-articular compared with intravenous tranexamic acid to reduce blood loss in primary total knee replacement: a double-blind, randomized, controlled, noninferiority clinical trial. J Bone Joint Surg Am. 2014;96(23):1937-44.

47. Patel JN, Spanyer JM, Smith LS, Huang J, Yakkanti MR, Malkani AL. Comparison of intravenous versus topical tranexamic acid in total knee arthroplasty: a prospective randomized study. J Arthroplasty. 2014;29(8): 1528-31.

48. Sarzaeem MM, Razi M, Kazemian G, Moghaddam ME, Rasi AM, Karimi M. Comparing efficacy of three methods of tranexamic acid administration in reducing hemoglobin drop following total knee arthroplasty. J Arthroplasty. 2014;29(8):1521-4

49. Soni A, Saini R, Gulati A, et al. Comparison between intravenous and intraarticular regimens of tranexamic acid in reducing blood loss during total knee arthroplasty. J Arthroplasty. 2014;29(8):1525-7.

50. Seo JG, Moon YW, Park SH, Kim SM, Ko KR. The comparative efficacies of intra-articular and IV tranexamic acid for reducing blood loss during total knee arthroplasty. Knee Surg Sports Traumatol Arthrosc. 2013;21(8):1869-74.

51. Maniar RN, Kumar G, Singhi T, Nayak RM, Maniar PR. Most effective regimen of tranexamic acid in knee arthroplasty: a prospective randomized controlled study in 240 patients. Clin Orthop Relat Res. 2012;470(9):2605-12.

52. Zhang S, Xie J, Cao G, Lei Y, Huang Q, Pei F. Six-dose intravenous tranexamic acid regimen further inhibits postoperative fibrinolysis and reduces hidden blood loss following total knee arthroplasty. J Knee Surg. 2019. https://doi.org/10.1055/s-0039-1694768. Epub ahead of print.

53. Chen Y, Chen Z, Cui S, Li Z, Yuan Z. Topical versus systemic tranexamic acid after total knee and hip arthroplasty: a meta-analysis of randomized controlled trials. Medicine (Baltimore). 2016:95(41):4656.

54. McCormack PL. Tranexamic acid: a review of its use in the treatment of hyperfibrinolysis. Drugs. 2012;72(5):585-617.

55. Xie J, Hu Q, Huang Q, Ma J, Lei Y, Pei F. Comparison of intravenous versus topical tranexamic acid in primary total hip and knee arthroplasty: an updated meta-analysis. Thromb Res. 2017;153:28-36.

56. Fu Y, Shi Z, Han B, et al. Comparing efficacy and safety of 2 methods of tranexamic acid administration in reducing blood loss following total knee arthroplasty: a meta-analysis. Medicine (Baltimore). 2016;95(50):5583.

57. Tzatzairis T, Drosos Gl, Vogiatzaki T, Tilkeridis K, Ververidis A, Kazakos K Multiple intravenous tranexamic acid doses in total knee arthroplasty without tourniquet: a randomized controlled study. Arch Orthop Trauma Surg. 2019;139(6):859-68.

58. Lei YT, Xie JW, Huang Q, Huang W, Pei FX. The antifibrinolytic and antiinflammatory effects of a high initial-dose tranexamic acid in total knee arthroplasty: a randomized controlled trial. Int Orthop. 2020;44(3):477-86.

59. Tammachote N, Raphiphan R, Kanitnate S. High-dose (3 g) topical tranexamic acid has higher potency in reducing blood loss after total knee arthroplasty compared with low dose (500 mg): a double-blind randomized controlled trial. Eur J Orthop Surg Traumatol. 2019;29(8):1729-35.

\section{Publisher's Note}

Springer Nature remains neutral with regard to jurisdictional claims in published maps and institutional affiliations.
Ready to submit your research? Choose BMC and benefit from:

- fast, convenient online submission

- thorough peer review by experienced researchers in your field

- rapid publication on acceptance

- support for research data, including large and complex data types

- gold Open Access which fosters wider collaboration and increased citations

- maximum visibility for your research: over $100 \mathrm{M}$ website views per year

At $\mathrm{BMC}$, research is always in progress.

Learn more biomedcentral.com/submission 